\title{
BOUNDARY VALUES AND THE TRANSFORMATION PROBLEM FOR CONSTANT PRINCIPAL STRAIN MAPPINGS
}

\author{
JULIAN GEVIRTZ
}

Received 17 April 2002

\begin{abstract}
We initiate a study of homeomorphisms $f$ with constant principal strains (cps) between smoothly bounded planar domains $D, D^{\prime}$. An initial result shows that in order for there to be such a mapping of a given Jordan domain $D$ onto $D^{\prime}$, a certain condition of an isoperimetric nature must be satisfied by the latter. Thereafter, we establish the fundamental fact that principal strain lines (characteristics) of such mappings necessarily have well-defined tangents where they meet $\partial D$. Using this, we obtain information about the boundary values of the Jacobian transformation of $f$, and finally we determine the class of all cps-homeomorphisms of a half-plane onto itself.
\end{abstract}

2000 Mathematics Subject Classification: 35L45, 35L60, 74B99, 30C35.

1. Introduction. This paper deals with mappings with constant principal strains, that is, mappings $f$ of a planar domain $D$ into $\mathbb{R}^{2}$ for which the principal stretches of the Jacobian matrix $J_{f}$ of $f$ at $z$ are distinct positive constants $m_{1}$ and $m_{2}$. Global properties of such $f$, to be referred to as $\left(m_{1}, m_{2}\right)$ mappings or less specifically as cps-mappings, were studied in [2, 3, 5, 6]. Homeomorphisms of this kind represent 2-dimensional deformations with constant principal strains such as those effected by cryptocrystalline solidification of a planar lamina, in which context the study of global properties of cps-mappings will yield, among other things, information about the manner in which such deformations can change shape as well as how the original mass can be shifted around in the process. In addition, $\left(m_{1}, m_{2}\right)$-mappings constitute a tractable subclass of $\left(m_{1}, m_{2}\right)$-quasi-isometries (i.e., local homeomorphisms for which the local stretching factors are merely constrained to lie between $m_{1}$ and $m_{2}$ ). Because they realize the local length change bounds extremally at all points, it is reasonable to believe that $\left(m_{1}, m_{2}\right)$-mappings manifest extremal behavior for some, at least, of the many open distortion questions for planar quasi-isometries (see [10, 11]).

Although, as we will presently explain, the systems of partial differential equations which define them are hyperbolic, significant analogies of conformal mappings discussed here and in the papers cited above lend credence to the belief that an interesting function theory for cps-mappings can ultimately be developed. Yet another motivation for this study lies in the fact that, other 
than conformal mappings, the classes of $\left(m_{1}, m_{2}\right)$-mappings are the only ones that can be defined by restricting the Jacobian to have values in a 2-parameter family of linear transformations in which no arbitrarily stipulated reference directions in the domain or image planes appear, a sine qua non for any direct extension to the more general Riemannian manifold context, as was pointed out in [2].

To continue this introductory discussion, it is advantageous to outline the formal local theory of $\left(m_{1}, m_{2}\right)$-mappings. Let $D \subset \mathbb{C}$ and let $f: D \rightarrow \mathbb{C}$ (we henceforth associate $\mathbb{R}^{2}$ with $\mathbb{C}$ and freely mix real and complex notation) be differentiable in $D$. In slight abuse of usual terminology, the nonnegative square roots $m_{1}(z), m_{2}(z)$ of the eigenvalues of the symmetric matrix $J_{f}(z)^{T} J_{f}(z)$ will be called the principal strains of $f$ at $z$. An $\left(m_{1}, m_{2}\right)$-mapping is an orientation-preserving mapping for which (the entries of) $J_{f}$ are in $\operatorname{Lip}(D)$, the class of locally Lipschitz continuous functions on $D$, and such that $m_{k}(z)=$ $m_{k}>0, k=1,2$ for all $z \in D$; the reason for the Lipschitz requirement will become clear in what follows. (See also the final paragraph of Section 7). We henceforth denote the set of all such mappings by the symbol cps $\left(D, m_{1}, m_{2}\right)$. It is immediate that for simply connected $D, f \in \operatorname{cps}\left(D, m_{1}, m_{2}\right)$ if and only if there are $\theta=\theta_{f}$ and $\phi=\phi_{f}$ in $\operatorname{Lip}(D)$ such that $J_{f}=T(-\phi) \sigma\left(m_{1}, m_{2}\right) T(\theta)$, where

$$
T(\theta)=\left[\begin{array}{cc}
\cos \theta & \sin \theta \\
-\sin \theta & \cos \theta
\end{array}\right], \quad \sigma\left(m_{1}, m_{2}\right)=\left[\begin{array}{cc}
m_{1} & 0 \\
0 & m_{2}
\end{array}\right]
$$

At each point $z \in D, e^{i \theta(z)}$ and $i e^{i \theta(z)}$ give the directions in which $f$ effects a length change by factors $m_{1}$ and $m_{2}$, respectively, and $e^{i \phi(z)}$ and $i e^{i \phi(z)}$ give the corresponding image directions. For $\theta \in \operatorname{Lip}(D)$, the integral curves of the direction fields $e^{i \theta(z)}$ and $i e^{i \theta(z)}$ will be called 1- and 2-characteristics, respectively. Straightforward calculations based on the equality of mixed secondorder partial derivatives show that if $D$ is simply connected and $\theta, \phi \in \operatorname{Lip}(D)$, then $T(-\phi) \sigma\left(m_{1}, m_{2}\right) T(\theta)$ is the Jacobian matrix of an $\left(m_{1}, m_{2}\right)$-mapping if and only if $m_{1} \theta-m_{2} \phi$ and $m_{2} \theta-m_{1} \phi$ are constant along each 1- and 2characteristic, respectively, that is, if and only if $\theta, \phi$ constitute a solution, in an appropriate sense, of the system

$$
D_{1}\left(m_{1} \theta-m_{2} \phi\right)=0 ; \quad D_{2}\left(m_{2} \theta-m_{1} \phi\right)=0,
$$

where $D_{1} u=\cos \theta u_{x}+\sin \theta u_{y}$ and $D_{2} u=-\sin \theta u_{x}+\cos \theta u_{y}$. Because of the obviously hyperbolic nature of this system, it is clear how we can manufacture large classes of cps-mappings as solutions to Cauchy or characteristic initial value problems. Further calculations show that a $C^{2}$-function $\theta$ is associated 
in this manner with an $\left(m_{1}, m_{2}\right)$-mapping if and only if it satisfies either of the second-order hyperbolic equations

$$
D_{2} D_{1} \theta=\left[D_{1} \theta\right]^{2} \quad \text { or } \quad D_{1} D_{2} \theta=-\left[D_{2} \theta\right]^{2} \text {. }
$$

For any $\theta$ satisfying these equations in $D$, the corresponding $\phi$ is determined to within an additive constant by (1.2). In the case of multiply connected $D$, of course, the $\phi$ so determined, and consequently the corresponding $f$, will in most cases be multiple-valued.

Equations (1.2), together with the appropriate weak counterpart of (1.3), to be explained in the following section, form the basis of the global theory of cps-mappings. The latter say exactly how the curvatures $D_{k} \theta$ of the characteristics change as we move along the orthogonal characteristics and imply, in particular, that the curvatures, unless initially 0 , must eventually blow up, that is, that $\theta$, and consequently $J_{f}$, must loose its local Lipschitz continuity if given enough room. Specifically, we have the sharp bound

$$
\left|D_{k} \theta\left(z_{0}\right)\right| \leq \frac{1}{\operatorname{dist}\left(z_{0}, \partial D\right)}
$$

(when the left-hand side is meaningful). This implies that $\operatorname{cps}\left(\mathbb{C}, m_{1}, m_{2}\right)$ contains only affine mappings, a cps-analogue of Liouville's theorem for analytic functions. More importantly, though, (1.4) tells us that $\left\{J_{f}: f \in \operatorname{cps}\left(D, m_{1}\right.\right.$, $\left.\left.m_{2}\right)\right\}$ is locally uniformly Lipschitz in $D$, and, in addition, allows us to derive a sharp distortion theorem (see [3]) to the effect that $f(N(z, r))$ is convex for all $f \in \operatorname{cps}\left(N(z, 1), m_{1}, m_{2}\right)$ if and only if $r \leq\left(\min \left\{m_{1}, m_{2}\right\} / \max \left\{m_{1}, m_{2}\right\}\right)^{2}$, where $N(z, r)$ denotes the disk of radius $r$ about $z$. This fact, together with the compactness principle (see Proposition 2.3), suggests that it should be possible to develop further sharp distortion results for cps-mappings which parallel some of the theorems of classical geometric function theory.

Instead of pursuing this possibility, however, in the present paper we examine the analogues of several other aspects of analytic function theory, all related to the general question, loosely referred to as the transformation prob$l e m$, of when and how the domains $D$ and $D^{\prime}$ can be mapped homeomorphically onto one another via cps-mappings. After Section 2, in which we formalize terminology and state the necessary basic facts, in Section 3 we take up the question as to whether two given domains $D$ and $D^{\prime}$ can be transformed onto one another by cps-mappings. Although for any Jordan domain $D$ $\cup_{m_{1}, m_{2}} \operatorname{cps}\left(D, m_{1}, m_{2}\right)$ is a very large class, we show that for any such smoothly bounded $D$ there are smoothly bounded $D^{\prime}$ onto which $D$ cannot be mapped homeomorphically by any cps-mapping; we do this by deriving a necessary condition on $D^{\prime}$, of an isoperimetric nature, for such a mapping to exist. Even though, in all likelihood, an intrinsic geometric characterization of all those 
$D^{\prime}$ onto which a given $D$ can be so transformed would be difficult to come by, the determination of all cps-mappings between two given domains, especially in the case where $D=D^{\prime}$, constitutes a significant issue. In order to pursue this question, however, it is necessary, in view of the hyperbolic nature of the basic equations, to determine to what extent such mappings are the solution of relevant Cauchy problems.

Fundamental to any analysis of this issue is the fact, established in Section 4, that if $f$ is any cps-homeomorphism of $D$ onto $D^{\prime}$, where $\partial D$ and $\partial D^{\prime}$ are smooth curves (i.e, with $C^{1}$ arc length parametrizations) and $C$ is a characteristic which meets $\partial D$ at $p$, then the limit of $\theta_{f}(z)$ as $z \rightarrow p$ along $C$ exists. This allows us to show in Section 5 that for each $z_{0} \in \partial D, \lim _{z \rightarrow z_{0}}\left(\theta_{f}(z), \phi_{f}(z)\right)$ exists in the sense that it tends to the solution of a "Riemann problem" for system (1.2) in a half-plane, so that the possibilities for the limiting behavior of $\theta_{f}(z)$ and $\phi_{f}(z)$ are governed by just two parameters (or four, if we include the inclinations of the tangents to $\partial D$ and $\partial D^{\prime}$ at $p$, and $f(p)$ ). This "boundary regularity" of $J_{f}$ is very much in the spirit of analogous results for the derivatives of conformal mappings such as the well-known theorem of Lindelöf ([12, Theorems 10.1, 10.4]).

It turns out that in the two significant cases of the half-plane and the exterior of a disk $\mathbb{C} \backslash N(0,1)$, geometric arguments based on (1.2) and (1.3) allow one to show that, apart from certain degeneracies which occasion no difficulties, all cps-self-homeomorphisms are associated with Cauchy problems, a circumstance which enables us to give a complete description of the relevant families; we do so for half-planes in Section 6 but leave the discussion of the somewhat more involved case of $\mathbb{C} \backslash N(0,1)$ to a subsequent paper. In both instances the reduction to Cauchy problems strongly depends on the fact that the geometry of the domains permits us to show that all characteristics meet $\partial D$ in exactly one point, a feature patently absent in the case, for example, of Jordan domains. In the concluding Section 7, we discuss some of the issues suggested in a natural way by the considerations of the preceding sections.

2. Preliminaries. Most of the facts stated in this section were proved in complete detail in [5], so that we will include proofs here only for points not discussed in that paper. To facilitate the discussion of characteristics, we begin by amplifying the notational conventions given in the introduction. As indicated, for $\theta \in \operatorname{Lip}(D)$, the complete integral curves of the direction fields $e^{i \theta(z)}$ and $i e^{i \theta(z)}$ will be called 1- and 2-characteristics, respectively, of $\theta$, or of $f$, when $\theta=\theta_{f}$. Note that if we regard $f$ as an $\left(m_{1}, m_{2}\right)$-mapping, rather than as an $\left(m_{2}, m_{1}\right)$-mapping, $e^{i \theta_{f}(z)}$ gives the direction in which lengths are changed by a factor of $m_{1}$ at $z$. Throughout, $\{i, j\}=\{1,2\}$. Arcs of $k$-characteristics will be called $k$-arcs or, less specifically, characteristic arcs. Unless otherwise stated, the term "characteristic" used alone refers to complete characteristics, which we sometimes emphasize by the inclusion of this modifier. When discussing an 
$i$-characteristic, the $j$-characteristics that cross it will be referred to as the $o r$ thogonal characteristics. If $z=z(s), \alpha_{1}<s<\alpha_{2}$ is a parametrization of a complete $k$-characteristic $C$, then for any $s_{0} \in\left(\alpha_{1}, \alpha_{2}\right), z\left(\left(\alpha_{1}, s_{0}\right]\right)$ and $z\left(\left[s_{0}, \alpha_{2}\right)\right)$ will be called $k$-half-characteristics. In addition, if $\lim _{s \rightarrow \alpha_{i}} z(s)=z_{0} \in \partial D, i=1$ or 2 , then we say that $C$ exits $D$ at $z_{0}$. Obviously, a characteristic can either not exit at all, exit at exactly one end, or exit at both ends (doubly exiting characteristic). When working with a characteristic $C$ parametrized in this way, we use the term to the right (left) to mean in the direction of $-i z^{\prime}(s)\left(i z^{\prime}(s)\right)$. The letter $s$ will always indicate an arc length parametrization.

With reference to a specific such $\theta$, a characteristic arc $C$ joining points $a, b \in$ $D$, oriented from $a$ to $b$, will be denoted by $a b$, and we use the abbreviation

$$
\Delta \theta(C)=\Delta \theta(a b)=\theta(b)-\theta(a)
$$

A domain $Q \subset D$ will be said to be a characteristic quadrilateral of $f$ if $\partial Q$ is a Jordan curve lying in $D$ and containing four points $a, b, c, d$ occurring in that order when $\partial Q$ is traversed (in either the positive or negative sense) and such that $a b$ and $c d$ are $i$-arcs, and $b c$ and $d a$ are $j$-arcs. We will refer to such a $Q$ as $a b c d$ and use the abbreviation

$$
\Delta^{2} \theta(a b c d)=\Delta \theta(b c)-\Delta \theta(a d)=\Delta \theta(d c)-\Delta \theta(a b) .
$$

Opposite sides of a characteristic quadrilateral will be referred to as translates of each other. For a given such $Q$, if $C_{k}$ is a $k$-arc joining opposite sides of $Q$ parametrized by $z_{k}(s), \tau_{k} \leq s \leq \sigma_{k}, k=1,2$, then the standard characteristic coordinate mapping $\zeta:\left[\tau_{i}, \sigma_{i}\right] \times\left[\tau_{j}, \sigma_{j}\right] \rightarrow Q$ maps $\left(s_{i}, s_{j}\right)$ onto the intersection of the $j$-characteristic through $z_{i}\left(s_{i}\right)$ with the $i$-characteristic through $z_{j}\left(s_{j}\right)$.

Let $D \subset \mathbb{C}$ be simply connected and let $\theta, \phi \in \operatorname{Lip}(D)$. Then, as indicated in the introduction, $J_{f}=T(-\phi) \sigma\left(m_{1}, m_{2}\right) T(\theta)$ is the Jacobian of a mapping if and only if (1.2) hold a.e., that is, if and only if

$$
R_{i}=m_{i} \theta-m_{j} \phi, \quad i=1,2,
$$

is constant along each $i$-characteristic. Moreover, $\theta \in \operatorname{Lip}(D)$ is $\theta_{f}$ for some $\left(m_{1}, m_{2}\right)$-mapping $f$ of $D$ if and only if $\Delta^{2} \theta(Q)=0$ for all characteristic quadrilaterals $Q$ of $\theta$ contained in $D$. Such functions $\theta$ are called HP-functions since the families of integral curves of the two fields $e^{i \theta}$ and $i e^{i \theta}$ for such $\theta$ are known as Hencky-Prandtl nets (HP-nets). (For the relevance of HP-nets to other contexts as well as a discussion of their elementary properties, see $[1,7,8,9,13]$.) We will refer to the fact that $\Delta^{2} \theta(Q)=0$ as the HP-property.

As indicated in the preceding section, $D_{1}$ and $D_{2}$ will denote differentiation with respect to arc length in the directions $e^{i \theta(z)}$ and $i e^{i \theta(z)}$, respectively. We use the symbol $\lambda_{k}(E), k=1,2$, to denote the $k$-dimensional measure of the set $E$, so that $\lambda_{1}(C)$, in particular, is the arc length of the simple arc $C$. 
Furthermore,

$$
N(X, r)=\cup\{N(z, r): z \in X\}
$$

Next, we explain the sense in which the blow-up equations (1.3) hold for general (i.e., not necessarily $C^{2}$ ) HP-functions $\theta$. We define $E_{i}=E_{i}(\theta)$ to be the set of all points $p$ such that if $z=z(s),-\epsilon<s<\epsilon$, with $z(0)=p$ is an arc length parametrization of an $i$-arc of $\theta$ containing $p$, then $\theta(z(s))$ is differentiable at $s=0$. Obviously, almost all points (with respect to arc length) of each $i$-characteristic belong to $E_{i}$ and almost all points of the domain on which $\theta$ is defined (with respect to $\lambda_{2}$ ) belong to $E_{1} \cap E_{2}$.

Proposition 2.1. Let $\theta$ be an HP-function on $D$ and let $C_{k}, k=1,2$ be the $k$-characteristic through $p \in E_{i}$. Then, $C_{j} \subset E_{i}$ and the relevant equation in (1.3) holds along $C_{j}$ when $D_{k}$ is interpreted as arc length differentiation along $C_{k}$ in the direction $e^{i \theta}$ for $k=1$ and $i e^{i \theta}$ for $k=2$.

If $z=z(s), \alpha<s<\beta$, is an arc length parametrization of an $m_{k}$-arc, then since $\theta \in \operatorname{Lip}(D), \kappa_{k}(z(s))=d \theta(z(s)) / d s=D_{k} \theta(z(s))$ exists almost everywhere on $(\alpha, \beta)$ and gives the curvature of the $k$-arc through $z(s)$. This means that if $\kappa_{i}(z)$ exists then $z$ is joined to $\partial D$ by a $j$-arc of length at most $1 /\left|\kappa_{i}(z)\right|$ emanating from the concave side of the $i$-characteristic through $z$. Because of this, we immediately obtain the following result.

Proposition 2.2. Let $f$ be a cps-mapping on $D$, then

$$
D_{k}^{+} \theta\left(z_{0}\right) \leq \frac{1}{\operatorname{dist}\left(z_{0}, \partial D\right)}
$$

for all $z_{0} \in D$, where

$$
D_{k}^{+} \theta(p)=\limsup _{z \rightarrow p}\left|D_{k} \theta(z)\right|
$$

From this, in turn, we easily deduce the following result.

Proposition 2.3 (compactness principle). Let $D$ and $B$ be a bounded domains, and let $\left\{f_{k}\right\}$ be a sequence of CPS mappings for which $f_{k}(D) \subset B$. Then $\left\{f_{k}\right\}$ contains a subsequence which converges uniformly to a cps-mapping $f$ on $D$, and for which the corresponding first-order derivatives converge locally uniformly on $D$ to those of $f$.

We will also need the following proposition.

Proposition 2.4. Let the mapping $\zeta: I_{1} \times I_{2} \rightarrow Q$, where $I_{i}=\left[\tau_{i}, \sigma_{i}\right]$, be a characteristic coordinate mapping (as described above). If the lengths of all of the translates of $C_{2}=z_{2}\left(I_{2}\right)$ along $C_{1}=z_{1}\left(I_{1} \times\left\{\tau_{2}\right\}\right)$ are at least $\rho$, then the area of $\zeta\left(I_{1} \times I_{2}\right)$ is at least $\rho \lambda_{1}\left(C_{1}\right) / 2$. 
Throughout, the term smooth curve will refer to one with a continuously turning tangent; that is, one for which the arc length parametrization is $C^{1}$. If $C$ is a characteristic of $f$ exiting $D$ at $p$ for which the limit of $\theta(z)$ exists as $z \rightarrow p$ (so that $C$ has a well-defined tangent at $p$ ), then it follows from (1.2) that the corresponding limit of $\phi(z)$ also exists, which means that $f(C)$ also has a well-defined tangent at $f(p)$.

Proposition 2.5. There exists a positive function $W=W(\mu, \alpha), \mu>1,0<$ $\alpha \leq \pi$, which for any given $\mu$ is nondecreasing in $\alpha$ and which has the following property. Let $D$ and $D^{\prime}$ be Jordan domains, and let $f$ be an $\left(m_{1}, m_{2}\right)$ homeomorphism of $D$ onto $D^{\prime}$. Let $E$ and $E^{\prime}$ be smooth arcs of $\partial D$ and $\partial D^{\prime}$. Let $C$ be a characteristic arc of $f$ which exits $D$ at endpoint $p$ of $E$ and for which the limit of $\theta_{f}(z)$ exists as $z \rightarrow p$ along $C$. Let $\alpha \in[0, \pi]$ be the angle formed by $C$ and $E$ at $p$, and let $\alpha^{\prime}$ denote the corresponding angle formed by $f(C)$ and $f(E)$ in the image. Let $m_{i}<m_{j}$. Then,

$$
\begin{aligned}
& \frac{m_{i}}{m_{j}} \alpha+W\left(\frac{m_{j}}{m_{i}}, \alpha\right) \leq \alpha^{\prime} \leq \frac{m_{j}}{m_{i}} \alpha \quad \text { if } C \text { is an } i \text {-characteristic, } \\
& \frac{m_{i}}{m_{j}} \alpha \leq \alpha^{\prime} \leq \frac{m_{j}}{m_{i}} \alpha-W\left(\frac{m_{j}}{m_{i}}, \alpha\right) \quad \text { if } C \text { is } j \text {-characteristic. }
\end{aligned}
$$

Proof. First assume that $C$ is an $i$-characteristic. Without loss of generality, we can assume that $p$ is the initial point of $E$. After appropriate compositions of $f$ with rigid motions, we can assume that $p=f(p)=0$, that the positively oriented tangents to both $E$ and $E^{\prime}$ at 0 have the direction of the positive real axis, and that the unit tangents to $C$ and $f(C)$ at 0 are $e^{i \alpha}$ and $e^{i \alpha^{\prime}}$. Let $\mu=$ $m_{j} / m_{i}$, and let $S(\gamma)$ denote the sector $\{z \mid 0<\arg z<\gamma\}$. Since $C$ is an $i$-arc, by applying the compactness principle to the family of mappings $f(n z) / m_{i} n$, we obtain $(1, \mu)$-homeomorphism $g$ of $S(\alpha)$ onto $S\left(\alpha^{\prime}\right)$ such that $g\left(t e^{i \alpha}\right)=t e^{i \alpha^{\prime}}$, $t \geq 0$. We have

$$
N(0,1) \cap S\left(\alpha^{\prime}\right) \subset g(N(0,1) \cap S(\alpha)),
$$

so that

$$
\frac{\alpha^{\prime}}{2}=\lambda_{2}\left(N(0,1) \cap S\left(\alpha^{\prime}\right)\right) \leq \lambda_{2}(g(N(0,1) \cap S(\alpha)))=\frac{\mu \alpha}{2},
$$

which gives the upper bound in (2.7). In addition,

$$
N\left(\frac{1+\mu}{2} e^{i \alpha}, \frac{\mu-1}{2}\right) \cap N(0,1)=\varnothing,
$$


and $S(\alpha)$ contains one of the semidisks $Q(\mu, \alpha)$ into which the line $\arg z=\alpha$ cuts the disk

$$
N\left(\frac{1+\mu}{2} e^{i \alpha}, \min \left\{\frac{\mu-1}{2 \mu}, \frac{1+\mu}{2} \sin \left(\min \left\{\alpha, \frac{\pi}{2}\right\}\right)\right\}\right) .
$$

Relation (2.11) implies that $Q(\mu, \alpha) \cap N(0,1)=\varnothing$. Since $g\left((1+\mu) e^{i \alpha} / 2\right)=$ $(1+\mu) e^{i \alpha^{\prime}} / 2$, it follows that $g(Q(\mu, \alpha)) \subset S\left(\alpha^{\prime}\right) \cap N(0, \mu)$. We have

$$
g(N(0,1) \cap S(\alpha)) \subset\left(S\left(\alpha^{\prime}\right) \cap N(0, \mu)\right) \backslash g(Q(\mu, \alpha)),
$$

so that

$$
\frac{\mu \alpha}{2}=\lambda_{2}(g(N(0,1) \cap S(\alpha))) \leq \frac{\alpha^{\prime} \mu^{2}}{2}-\mu \lambda_{2}(Q(\mu, \alpha))
$$

that is, $\alpha \leq \mu \alpha^{\prime}-2 \lambda_{2}(Q(\mu, \alpha))$, so that we have

$$
\frac{\alpha}{\mu}+W_{1}(\mu, \alpha) \leq \alpha^{\prime} \leq \mu \alpha
$$

where

$$
W_{1}(\mu, \alpha)=\frac{\pi(\min \{(\mu-1) / 2 \mu,(1+\mu) / 2 \sin (\min \{\alpha, \pi / 2\})\})^{2}}{\mu} .
$$

If $C$ is a $j$-characteristic, then we apply (2.15) to the $\left(1 / m_{1}, 1 / m_{2}\right)$-homeomorphism $f^{-1}$ to obtain

$$
\alpha^{\prime} \leq \mu \alpha-\mu W_{1}\left(\mu, \alpha^{\prime}\right), \quad \alpha^{\prime} \geq \frac{\alpha}{\mu}
$$

Since for fixed $\mu, W_{1}(\mu, \alpha)$ is nondecreasing in $\alpha$, it follows that $W_{1}\left(\mu, \alpha^{\prime}\right) \geq$ $W_{1}(\mu, \alpha / \mu)$. Thus, in the case that $C$ is a $j$-characteristic, we have

$$
\frac{\alpha}{\mu} \leq \alpha^{\prime} \leq \mu \alpha-\mu W_{1}\left(\mu, \frac{\alpha}{\mu}\right)
$$

so that Proposition 2.5 is established with

$$
W(\mu, \alpha)=\min \left\{W_{1}(\mu, \alpha), \mu W_{1}\left(\mu, \frac{\alpha}{\mu}\right)\right\},
$$

which is clearly a positive nonincreasing function of $\alpha$. 
Proposition 2.6. There is a positive decreasing function $\gamma=\gamma_{m_{1}, m_{2}}(t)$ with the following property: let $D$ and $D^{\prime}$ be Jordan domains and let $f: D \rightarrow D^{\prime}$ be an $\left(m_{1}, m_{2}\right)$-homeomorphism of $D$ onto $D^{\prime}$ under which smooth boundary arcs $E$ and $E^{\prime}$ correspond to each other. Let $C$ be a characteristic of $f$ joining the endpoints $a$ and $b$ of $E$ for which the limits of $\theta(z)$ exist as $z \rightarrow a, b$ along $C$, and for which the interior angles of the simple closed curve $E \cup C$ at $a$ and $b$ are $\alpha$ and $\beta$. Let the change in the tangent inclinations along $E$ and $E^{\prime}$ (measured in the positive direction along the boundaries) be $d$ and $d^{\prime}$. Then, $|d|+\left|d^{\prime}\right| \geq$ $\gamma(\alpha)+\gamma(\beta)$.

Proof. Let $m_{i}<m_{j}$ and let $C$ be an $i$-characteristic taken as positively oriented with respect to the interior of $E \cup C$. Let $\mu=m_{j} / m_{i}$. Then,

$$
\begin{gathered}
\Delta \theta(C)+d=\alpha+\beta, \\
\frac{\Delta \theta(C)}{\mu}+d^{\prime}=\Delta \phi(C)+d^{\prime}=\alpha^{\prime}+\beta^{\prime} \geq \frac{\alpha+\beta}{\mu}+W(\mu, \alpha)+W(\mu, \beta),
\end{gathered}
$$

where $\alpha^{\prime}$ and $\beta^{\prime}$ are the image angles corresponding to $\alpha$ and $\beta$, and $W$ is the function of the preceding proposition defined in (2.16) and (2.19). Thus

$$
\frac{\alpha+\beta-d}{\mu}+d^{\prime} \geq \frac{\alpha+\beta}{\mu}+W(\mu, \alpha)+W(\mu, \beta)
$$

so that

$$
|d|+\left|d^{\prime}\right| \geq d^{\prime}-\frac{d}{\mu} \geq W(\mu, \alpha)+W(\mu, \beta) .
$$

A similar bound holds in the case that $C$ is a $j$-characteristic.

An examination of the proof (with $d=d^{\prime}=0$ ) shows that under the same hypotheses, there cannot exist a characteristic which joins an interior point of $E$ to itself.

Proposition 2.7. There is a $\tau_{0}=\tau_{0}\left(m_{i}, m_{j}\right)>0$ with the following property: let $D, D^{\prime}, E, E^{\prime}$, and $f$ be as in the preceding proposition. Let $a, b$, and $c$ be interior points of E. Let $C$ be an i-arc which joins $a$ and $b$ and let $\bar{C}$ be $a$ $j$-arc joining a point e of $C$ to $c$ outside the subdomain $U$ of $D$ bounded by $C$ and the arc $a b$ of $E$. Assume that the limits of $\theta(z)$ exist as $z \rightarrow a$ along $C$ and as $z \rightarrow c$ along $\bar{C}$. Let the interior angle of $\partial U$ at $a$ be $\alpha \geq 0$ and let $\Delta \theta(a e)=\delta$. If $\alpha,|\delta|<\tau_{0}$, then $|d|+\left|d^{\prime}\right| \geq \tau_{0}$, where $d$ and $d^{\prime}$ are the changes in the angle tangent to $\partial D$ and $\partial D^{\prime}$ along $a c$ and $f(a c)$, respectively.

Proof. Let $T$ be the curvilinear triangle bounded by the arc ae of $C, \bar{C}$, and the subarc $B$ of $E$ joining $a$ and $c$. Let $\gamma \geq 0$ and $\omega$ be the interior angles of 
$T$ at $c$ and $e$, respectively. There are two cases: (i) $\omega=3 \pi / 2$ and (ii) $\omega=\pi / 2$, corresponding, respectively, to the cases that $a$ comes before and after $c$ when $E$ is traversed in the positive sense with respect to $D$. Let $\delta$ and $\bar{\delta}$ be the changes in $\theta$ along ae and $\bar{C}$ when these arcs are traversed in the positive sense with respect to $T$, and let $d$ and $d^{\prime}$ be the corresponding changes in the tangent angle along $B$ and $f(B)$. Let $\mu=m_{j} / m_{i}$ and let $\alpha^{\prime}$ and $\gamma^{\prime}$ be the angles corresponding to $\alpha$ and $\gamma$ in $f(T)$. In case (i) we have

$$
\bar{\delta}=\frac{\pi}{2}+\gamma+\alpha-\delta-d, \quad \mu \bar{\delta}=\frac{\pi}{2}+\gamma^{\prime}+\alpha^{\prime}-\frac{1}{\mu} \delta-d^{\prime}
$$

Let $\alpha,|\delta|<\tau_{0}$. Assume first that $\mu>1$. From Proposition 2.5 and the second of these equations it follows that

$$
\mu \bar{\delta} \leq \frac{\pi}{2}+\mu \gamma+\mu \alpha-\frac{1}{\mu} \delta-d^{\prime}
$$

so that in light of the first equation,

$$
\begin{aligned}
\mu \bar{\delta} & \leq \frac{\pi}{2}+\mu\left(\bar{\delta}-\frac{\pi}{2}-\alpha+\delta+d\right)+\mu \alpha-\frac{1}{\mu} \delta-d^{\prime} \\
& \leq(1-\mu) \frac{\pi}{2}+\mu \bar{\delta}+2 \mu \tau_{0}+\mu\left(|d|+\left|d^{\prime}\right|\right)
\end{aligned}
$$

so that $|d|+\left|d^{\prime}\right| \geq\left((\mu-1)(\pi / 2)-2 \mu \tau_{0}\right) / \mu$, which gives the desired conclusion with an appropriate $\tau_{0}$. If $\mu<1$, then we have

$$
\begin{aligned}
\mu \bar{\delta} & \geq \frac{\pi}{2}+\mu \gamma+\mu \alpha-\frac{1}{\mu} \delta-d^{\prime} \\
& =\frac{\pi}{2}+\mu\left(\bar{\delta}-\frac{\pi}{2}-\alpha+\delta+d\right)+\mu \alpha-\frac{1}{\mu} \delta-d^{\prime} \\
& \geq(1-\mu) \frac{\pi}{2}+\mu \bar{\delta}-\frac{2}{\mu} \tau_{0}-\left(|d|+\left|d^{\prime}\right|\right)
\end{aligned}
$$

so that $|d|+\left|d^{\prime}\right| \geq(1-\mu)(\pi / 2)-(2 / \mu) \tau_{0}$, which again gives the desired result. Case (ii) is handled similarly; here we have

$$
\bar{\delta}=\frac{\pi}{2}+\gamma-\alpha-\delta-d, \quad \mu \bar{\delta}=\frac{\pi}{2}+\gamma^{\prime}-\alpha^{\prime}-\frac{1}{\mu} \delta-d^{\prime}
$$

Assume first that $\mu>1$. From Proposition 2.5 and the second of these equations it follows that

$$
\mu \bar{\delta} \leq \frac{\pi}{2}+\mu \gamma-\frac{1}{\mu} \delta-d^{\prime}
$$


so that in light of the first equation,

$$
\begin{aligned}
\mu \bar{\delta} & \leq \frac{\pi}{2}+\mu\left(\bar{\delta}-\frac{\pi}{2}+\alpha+\delta+d\right)-\frac{1}{\mu} \delta-d^{\prime} \\
& \leq(1-\mu) \frac{\pi}{2}+\mu \bar{\delta}+3 \mu \tau_{0}+\mu\left(|d|+\left|d^{\prime}\right|\right),
\end{aligned}
$$

so that $|d|+\left|d^{\prime}\right| \geq\left((\mu-1)(\pi / 2)-3 \mu \tau_{0}\right) / \mu$, which again gives the desired conclusion. Finally, if $\mu<1$, we have

$$
\begin{aligned}
\mu \bar{\delta} & \geq \frac{\pi}{2}+\mu\left(\bar{\delta}-\frac{\pi}{2}+\alpha+\delta+d\right)-\frac{1}{\mu} \alpha-\frac{1}{\mu} \delta-d^{\prime} \\
& \geq(1-\mu) \frac{\pi}{2}+\mu \bar{\delta}-\frac{3}{\mu} \tau_{0}-\left(|d|+\left|d^{\prime}\right|\right),
\end{aligned}
$$

so that $|d|+\left|d^{\prime}\right| \geq(1-\mu)(\pi / 2)-(3 / \mu) \tau_{0}$, and we are done.

Proposition 2.8. Let $D$ be a Jordan domain, and let $z=z(s), \alpha<s<\beta$ be an arc length parametrization of a full characteristic $C$ of an HP-net on D. Then, $\lim _{z \rightarrow \alpha} z(s)$ and $\lim _{z \rightarrow \beta} z(s)$ exist and belong to $\partial D$.

Proof. First of all, $\operatorname{dist}(z(s), \partial D) \rightarrow 0$ as $s \rightarrow \alpha$ (and similarly as $s \rightarrow \beta$ ). If this were not true, then there would be a $z_{0} \in D$ and an $\epsilon>0$ such that for some sequence $\left\{s_{i}\right\}$ tending to $\alpha, z\left(s_{i}\right) \rightarrow z_{0}$ but $z\left(\left[s_{i}, s_{i+1}\right]\right) \cap \partial N\left(z_{0}, \epsilon\right) \neq \varnothing$. But from this it would follow that some orthogonal characteristic crosses $C$ twice, an impossible occurrence in light of the simple connectivity of $D$. We can now show that, in fact, $z(s) \rightarrow a, b \in \partial D$ as $s \rightarrow \alpha, \beta$, respectively. Assume that this is not so, as $s \rightarrow \beta$, for example. The foregoing then implies that there is an $\operatorname{arc} E$ of $\partial D$, each point of which is an accumulation point of $C_{\gamma}=\{z(s): s>\gamma\}$ for each $\gamma \in(\alpha, \beta)$. Since in this case $C$ is clearly not a straight line segment, it follows from the comment immediately following Proposition 2.1 that there is an orthogonal half-characteristic $C^{\prime}$ of finite length which joins some $z(\sigma)$ to a point $e \in \partial D$. Since $C$ cannot cross $C^{\prime}$ twice in $D, C_{\sigma} \subset D \backslash C^{\prime}$. Let $z_{1}, z_{2}$ be distinct points of $E \backslash\{e\}$. For each $\delta>0, C_{\sigma}$ has a subarc $p p^{\prime} \subset N(\partial D, \delta) \backslash C^{\prime}$, with $p, p^{\prime} \in N\left(z_{1}, \delta\right)$ and a point $p^{\prime \prime} \in p p^{\prime} \cap N\left(z_{2}, \delta\right)$. For obvious topological reasons, for each sufficiently small $\delta$, there must be a point $q$ on $p p^{\prime}$ which is joined to a point in $N(z, \delta)$ by an orthogonal characteristic $\operatorname{arc} B$ of length at least $\left|z_{1}-z_{2}\right|-2 \delta$ such that the curvature of $C$ at $q$ tends to infinity as $\delta \rightarrow 0$ and $C$ is concave towards the side from which $B$ emanates. But this clearly violates Proposition 2.1, as indicated in the paragraph immediately following its statement.

Proposition 2.9. If $D$ is a Jordan domain with smooth boundary, then all characteristics of every HP-net on D have finite length. 
Proof. Let $C$ be parametrized as in the statement of the preceding lemma and let $b=\lim _{z \rightarrow \beta} z(s)$. Without loss of generality we assume that $b=0$ and that the positively oriented tangent to $\partial D$ at 0 has the direction of the positive real axis. We only consider the part of $C^{+}=z([(\alpha+\beta) / 2, \beta))$ that is within $\epsilon$ of $b$, where $|z((\alpha+\beta) / 2)-b| \geq 2 \epsilon$ and $\epsilon$ is so small that $N(0,2 \epsilon) \cap D$ is so close to being a semidisk that for $0<\delta \leq 2 \epsilon, \partial N(0, \delta) \cap D$ is connected and $\operatorname{dist}(\delta i, \partial D) \geq \delta / 2$. Now consider any $\delta \leq \epsilon$ and look at the part $C_{\delta}=C^{+} \cap\{z$ : $\Re z>0,(1 / 2) \delta<|z|<\delta\}$ of $C^{+}$in the right half of the "semiring" of points of $D$ within $(1 / 2) \delta$ and $\delta$ of $b$. Note that $C_{\delta}$ may have several components. For each $p \in C_{\delta}$ let $E_{p}$ denote the maximal orthogonal characteristic arc in $N(0,2 \delta) \cap D$ emanating rightwards from $p$ (as we move along $C$ towards $b$ ). By our choice of $\epsilon, \lambda_{1}\left(E_{p}\right) \geq(1 / 4) \delta$. In addition, all of the $E_{p}$ are disjoint. It then follows from the lower bound for the area of characteristic quadrilaterals given in Proposition 2.4 that $4 \pi \delta^{2}=\lambda_{2}(N(0,2 \delta)) \geq(1 / 8) \delta \lambda_{1}\left(C_{\delta}\right)$, so that $\lambda_{1}\left(C_{\delta}\right) \leq$ $32 \pi \delta$. Obviously, the same bound will hold when the left half of the semiring is considered instead of the right half. But by considering $\delta=(1 / 2)^{n} \epsilon, n \geq 0$, we will then have that the length of the part of $C^{+}$within $\epsilon$ of $b$ is at most $128 \pi \epsilon$. This shows that $\lambda_{1}\left(C^{+}\right)<\infty$. By symmetry, $\lambda_{1}(C)<\infty$.

3. A property of image domains. Let $D$ be a simply connected domain. Because an $\left(m_{1}, m_{2}\right)$-homeomorphism can alter lengths of curves only by factors between $m_{1}$ and $m_{2}$, it is obviously not possible to transform $D$ onto every other simply connected domain by means of a homeomorphism in cps $\left(D, m_{1}\right.$, $m_{2}$ ). Furthermore, because of the local Lipschitz continuity of such $f$, cps-homeomorphisms necessarily preserve both smoothness and irregularity of the boundary to some extent. It is, however, not unreasonable to ask if each smoothly bounded Jordan domain $D$ can be transformed by some cpshomeomorphism, with appropriate $m_{1}, m_{2}$ onto any other such domain. We show that cps-mappings, while clearly forming a "large" class, do not possess this transformation capability; indeed, the following theorem shows that all homeomorphic cps-images of a smoothly bounded Jordan domain $D$ must satisfy a shape requirement of an isoperimetric nature. In the following theorem, $\iota(E)=\sup \{r: N(z, r) \subset E\}$, the inradius of the Jordan domain $E$. It is clear that there is a $z_{0}$ in $E$ such that $N\left(z_{0}, \iota(E)\right) \subset E$.

THEOREM 3.1. For each smoothly bounded Jordan domain $D$, there exists a constant $C=C_{D}$ such that $\lambda_{1}(\partial E) \leq C \lambda_{2}(E) / \iota(E)$ for every Jordan domain $E$ which is the image of $D$ under a cps-homeomorphism.

Proof. Let $g \in \operatorname{cps}\left(N\left(z_{0}, \rho\right), m_{1}, m_{2}\right)$. It follows from Proposition 2.2 that for $0<\xi<1, \theta_{g}$ is Lipschitz continuous with Lipschitz constant $(\rho(1-\xi))^{-1}$ in the concentric disk $N\left(z_{0}, \xi \rho\right)$. Upon taking into account what this says about the curvature of characteristics of such $g$, we see that for very small $\xi$ characteristic arcs in $N\left(z_{0}, \xi \rho\right)$ are "virtually" straight line segments, so that there 
is a $\xi_{0}$ such that any two distinct $i$-arcs $a_{1} b_{1}$ and $a_{2} b_{2}$ of $g$ in $N\left(z_{0}, \rho\right)$ with $a_{1}, b_{1}, a_{2}, b_{2} \in \partial N\left(z_{0}, 2 \xi_{0} \rho\right)$ may be joined by a $j$-arc of $g$ lying entirely in $N\left(z_{0}, \rho\right)$. From this it follows that if $F \supset N\left(z_{0}, \rho\right)$ is any simply connected domain and $g \in \operatorname{cps}(F)$ and $A$ is any $i$-arc of $g$ in $F$, then $A \cap N\left(z_{0}, 2 \xi_{0} \rho\right)$ has at most one component. Indeed, were it to have more than one, then by the foregoing these could be joined by a $j$-arc $B$ of $g$ in $N\left(z_{0}, \rho\right)$. But then $B$ and $A$ would have at least two points of intersection, which is incompatible with the simple connectedness of $F$. By making $\xi_{0}$ smaller, if necessary, we can assume that all $i$-arcs $a b$ in $N\left(z_{0}, \xi_{0} \rho\right)$ are contained in $N\left([a, b], \xi_{0} \rho / 100\right)$, so that for any $m<\xi_{0} \rho / 100$ and any $i$-arc $A$,

$$
\lambda_{2}\left(N(A, m) \cap N\left(z_{0}, \xi_{0} \rho\right)\right) \leq 10 \xi_{0} \rho m .
$$

If $m \geq \xi_{0} \rho / 100$, then clearly we have

$$
\lambda_{2}\left(N(A, m) \cap N\left(z_{0}, \xi_{0} \rho\right)\right) \leq \pi\left(\xi_{0} \rho\right)^{2}<C_{0} \xi_{0} \rho m,
$$

with $C_{0}=100 \pi$, so that (3.2) holds for all $m, \rho$. These numbers $\xi_{0}$ and $C_{0}$ are universal constants, that is, (3.2) is valid for all $\rho>0$ and all $i$-arcs $A$ of any cps-mapping of any domain $F$ which contains a disk $N\left(z_{0}, \rho\right)$.

Obviously, there is a cps-homeomorphism of $D$ onto $E$ if and only if there is one onto $F=\sqrt{\lambda_{2}(D) / \lambda_{2}(E)} E$. We show that there is a constant $C^{\prime}$ such that if $F$ is a cps-homeomorphic image of $D$, for which

$$
F \supset N\left(z_{0}, r\right), \quad \lambda_{2}(F)=\lambda_{2}(D),
$$

then $\lambda_{1}(\partial F) \leq C^{\prime} / r$. The desired result follows with $C_{D}=C^{\prime} / \lambda_{2}(D)$.

Let $D^{\prime}$ be any fixed Jordan subdomain of $D$ for which $\overline{D^{\prime}} \subset D$ and such that $\lambda_{2}\left(D \backslash D^{\prime}\right)<\pi\left(\xi_{0} r / 2\right)^{2}$. On the basis of the a priori bound on the Lipschitz constants for $\theta$ mentioned at the beginning of the first paragraph of the proof, it is easy to see that there exists some integer $K$ such that for each $f \in \operatorname{cps}(D)$, there is a set $\left\{C_{1}, C_{2}, \ldots, C_{l}\right\}$ of $l \leq K j$-arcs of $f$ with the property that each point of $D^{\prime}$ can be joined to some point of their union by an $i$-arc of $f$ of length at most 1 . We stress that $K$ depends only on $D^{\prime}$, and so is fixed, since $D^{\prime}$ is. The arcs $C_{1}, C_{2}, \ldots, C_{l}$ themselves, of course, depend on $f \in \operatorname{cps}(D)$.

Let $f$ be an $\left(m_{i}, m_{j}\right)$-homeomorphism of $D$ with $m_{i}<m_{j}$, for which $F=$ $f(D)$ satisfies (3.3). It follows from the foregoing that all points of $f\left(D^{\prime}\right)$ are within $m_{i}$ of $\bigcup_{k=1}^{l} f\left(C_{k}\right)$, that is, that

$$
f\left(D^{\prime}\right) \subset \bigcup_{k=1}^{l} N\left(f\left(C_{k}\right), m_{i}\right) .
$$


Since $\lambda_{2}(F)=\lambda_{2}(D)$, it follows that $m_{i} m_{j}=1$, so that

$$
\lambda_{2}\left(N\left(z_{0}, \xi_{0} r\right) \backslash f\left(D^{\prime}\right)\right) \leq \lambda_{2}\left(F \backslash f\left(D^{\prime}\right)\right)=\lambda_{2}\left(D \backslash D^{\prime}\right)<\frac{\pi\left(\xi_{0} r\right)^{2}}{4} .
$$

Thus,

$$
\lambda_{2}\left(N\left(z_{0}, \xi_{0} r\right) \cap f\left(D^{\prime}\right)\right) \geq \frac{3 \pi}{4}\left(\xi_{0} r\right)^{2}
$$

Now, for each $k, A=f\left(C_{k}\right)$ is a characteristic arc of $g=f^{-1}$ in $F$, so that (3.2) holds with $\rho=r$ and $m=m_{i}$. But then by (3.4) and (3.6), $3 \pi\left(\xi_{0} r\right)^{2} / 4 \leq$ $C_{0} K \xi_{0} m_{i} r$, so that $m_{i} \geq 3 \pi \xi_{0} r / 4 C_{0} K$, and therefore, since $m_{i} m_{j}=1, m_{j} \leq$ $4 C_{0} K / 3 \pi \xi_{0} r$. Since $f$ increases lengths at most by a factor of $m_{j}$, this bound implies that $\lambda_{1}(\partial F)=\lambda_{1}(f(\partial D)) \leq C^{\prime} / r$, where $C^{\prime}=\left(4 C_{0} K / 3 \pi \xi_{0}\right) \lambda_{1}(\partial D)$. This finishes the proof of the theorem.

4. Tangents to characteristics at boundary points. The main result of this section, Theorem 4.1, tells us that any characteristic of a cps-homeomorphism of a smoothly bounded domain $D$ onto another such domain that meets $\partial D$ does so at a well-defined angle. This is the key to most of what is to follow since without knowledge of this fact we would, in effect, be limited to studying the transformation problem for cps-mappings within the confines of artificially imposed a priori hypotheses about regularity at the boundary.

Let $D$ and $D^{\prime}$ be Jordan domains, and let $f$ be an $\left(m_{1}, m_{2}\right)$-homeomorphism of $D$ onto $D^{\prime}$. The mapping $f$ obviously has a unique continuous extension to a homeomorphism of $\bar{D}$ onto $\overline{D^{\prime}}$. Let $K$ and $K^{\prime}$ be open $C^{1} \operatorname{arcs}$ of $\partial D, \partial D^{\prime}$ which correspond to each other under $f$. Let $C$ be a characteristic arc of $f$ which joins a point of $D$ to $p \in K$. It easily follows from Proposition 2.9 that $\lambda_{1}(C)<\infty$. Let $C$ be parametrized by $z=w(s), 0 \leq s \leq \sigma_{0}$, where $w\left(\sigma_{0}\right)=p$. For notational convenience we may assume, without loss of generality, that $p=f(p)=0$, and that the positively oriented tangents to both $\partial D$ and $\partial D^{\prime}$ at 0 point in the direction of the positive $x$-axis. Under these assumptions we will prove the following result.

THEOREM 4.1. The limits of $\theta(w(s))$ and $\phi(w(s))$ as $s \rightarrow \sigma_{0}$ exist.

Examples can be constructed to show that the smoothness of $K^{\prime}$ is necessary. Before beginning the proof, we establish the following lemma.

LEMMA 4.2. If $\lim _{s \rightarrow \sigma_{0}} \theta(w(s))$ does not exist, then there is some $\xi>0$ such that for all $\sigma<\sigma_{0}$ there are numbers $s_{1}<s_{2}$ and $s_{1}^{\prime}<s_{2}^{\prime}$ in $\left(\sigma, \sigma_{0}\right)$ such that

$$
\Delta \theta\left(w\left(s_{1}\right) w\left(s_{2}\right)\right) \geq \xi, \quad \Delta \theta\left(w\left(s_{1}^{\prime}\right) w\left(s_{2}^{\prime}\right)\right) \leq-\xi
$$


Proof. If (4.1) does not hold for any $\xi>0$, then either

$$
\limsup _{s_{1}, s_{2} \rightarrow \sigma_{0}} \Delta \theta\left(w\left(s_{1}\right) w\left(s_{2}\right)\right) \leq 0 \quad \text { or } \quad \liminf _{s_{1}, s_{2} \rightarrow \sigma_{0}} \Delta \theta\left(w\left(s_{1}\right) w\left(s_{2}\right)\right) \geq 0
$$

where it is understood that $s_{1}<s_{2}$. If, for example, the upper limit is nonpositive, then the only way $\lim _{s \rightarrow \sigma_{0}} \theta(w(s))$ could fail to exist would be for $\theta(w(s))$ to tend to $-\infty$, in which case the curve $C$ would ultimately be an infinite (inward) clockwise spiral when traversed in the direction of increasing $s$, which is incompatible with the hypothesis that $w(s) \rightarrow p \in \partial D$. In the case of a nonnegative lower limit we arrive at the same contradiction with a counterclockwise spiral.

Proof of Theorem 4.1. Let $C$ be an $i$-characteristic. To prove the theorem, it is enough to obtain a contradiction from the assumption that the limit of $\theta(w(s))$ does not exist since the existence of that of $\phi(w(s))$ will then follow from (1.2). Furthermore, it is sufficient to assume that $m_{i}>m_{j}$ since the truth of the theorem in the opposite case will follow from consideration of $f^{-1}$.

The curvature $\kappa(s)=d \theta(w(s)) / d s$ exists a.e. on $\left(0, \sigma_{0}\right)$. It follows immediately from Lemma 4.2 and the fact that $\sigma_{0}<\infty$ that for each $k>0$ and each $\sigma<\sigma_{0}$

$$
\lambda_{1}\left(\left\{s \in\left(\sigma, \sigma_{0}\right): \kappa(s) \geq k\right\}\right)>0, \quad \lambda_{1}\left(\left\{s \in\left(\sigma, \sigma_{0}\right): \kappa(s) \leq-k\right\}\right)>0 .
$$

Now, for each $s \in\left(0, \sigma_{0}\right)$ we denote by $C_{s}$ the entire open $j$-characteristic arc passing through $w(s)$. In addition, we denote by $C_{s}^{+}$and $C_{s}^{-}$the $j$-halfcharacteristics emanating rightwards and leftwards from $w(s)$, respectively. These half-characteristics are considered to contain $w(s)$ (and so are halfopen). On both $C_{s}^{+}$and $C_{s}^{-}$we consider the positive direction to be that which corresponds to movement away from $C$. Now, Proposition 2.1 tells us that at each point $s \in\left(0, \sigma_{0}\right)$ for which $\kappa(s)$ exists,

$$
\lambda_{1}\left(C_{s}^{-}\right) \leq \frac{1}{\kappa(s)}, \quad \text { if } \kappa(s)>0, \quad \lambda_{1}\left(C_{s}^{+}\right) \leq-\frac{1}{\kappa(s)} \quad \text { if } \kappa(s)<0 .
$$

From this, the fact that by $(4.3) \kappa\left(s_{j}\right) \rightarrow+\infty$ and $\kappa\left(s_{j}^{\prime}\right) \rightarrow-\infty$ for appropriate sequences $\left\{s_{j}\right\},\left\{s_{j}^{\prime}\right\}$ tending to $\sigma_{0}$ and the fact that distinct $j$-characteristics cannot intersect in $D$, it follows that

$$
\lim _{S \rightarrow \sigma_{0}} \operatorname{diam}\left(C_{s}\right)=0
$$

Now let $s_{0}>0$ be a density point of $\{s: \kappa(s)>0\}$. Let $0<\xi<\min \left\{s_{0}, \sigma_{0}-s_{0}\right\}$ be such that

$$
\lambda_{1}\left(\left\{s \in\left(s_{0}-\xi, s_{0}+\xi\right): \kappa(s)>0\right\}\right)>\frac{3 \xi}{2} .
$$


Since by (4.3) $s_{0}$ can be chosen arbitrarily close to $\sigma_{0}$, it follows from (4.5) that we may in addition assume that

$$
\bigcup\left\{C_{s}: s_{0}-\xi \leq s \leq s_{0}+\xi\right\} \subset N(p, R),
$$

where $N(p, R) \cap \partial D \subset K$. Let $z=v(\bar{s}), 0 \leq \bar{s}<\rho$, be the arc length parametrization of $C_{s_{0}}^{+}$with $v(0)=w\left(s_{0}\right)$. For $\tau>0$ sufficiently small, the characteristic quadrilateral consisting of the translates of the $i$-arc $w\left(\left[s_{0}-\xi, s_{0}+\xi\right]\right)$ along the $j$-arc $v([0, \tau))$ exists, that is, lies entirely in $D$. Let $\tau_{1}=\sup \{\tau$ : $Q_{\tau}$ exists $\}$. Let $\zeta(s, \bar{s})$ give the standard characteristic coordinate mapping of $\left[s_{0}-\xi, s_{0}+\xi\right] \times\left[0, \tau_{1}\right]$ onto $Q_{\tau_{1}}$. It follows from (4.6) that for $\tau<\tau_{1}$, the lengths of the translates of $w\left(\left[s_{0}-\xi, s_{0}\right]\right)$ and of $w\left(\left[s_{0}, s_{0}+\xi\right]\right)$ along $C_{s_{0}}$ down to $v(\tau)$ are at least $\xi / 2$. From Proposition 2.8 it follows that $\zeta\left(s, \tau_{1}\right)=\lim _{\tau \rightarrow \tau_{1}} \zeta(s, \tau)$ exists for each $s \in w\left(\left[s_{0}-\xi, s_{0}+\xi\right]\right)$. But then for some $s_{1}, \zeta\left(s_{1}, \tau_{1}\right) \in \partial D$, since otherwise, by compactness, $\zeta\left(\left[s_{0}-\xi, s_{0}+\xi\right], \tau_{1}\right) \subset D$, which contradicts the definition of $\tau_{1}$. If $s_{0}-\xi \leq s_{1} \leq s_{0}$, then at all points at which it exists the curvature of the characteristic arc $\zeta\left(s_{1},\left[0, \tau_{1}\right]\right.$ ) (with the positive sense corresponding to movement away from $C$ ) is bounded above by $2 / \xi$, by what was pointed out above about the length of the translates of $w\left(\left[s_{0}, s_{0}+\xi\right]\right)$. Similarly, if $s_{0} \leq s_{1} \leq s_{0}+\xi$, then this curvature is bounded below by $-2 / \xi$. From Lemma 4.2 (together with its consequence (4.3)), it therefore follows that if $s_{0}$ is a density point of $\{s: \kappa(s)>0\}$ for which (4.6) and (4.7) hold, then there are points $s_{1}$ arbitrarily close to $s_{0}$ for which the limit of $\theta(z)$ as $z$ approaches $\partial D$ along $C_{s_{1}}^{+}$exists. Obviously, the analogous statement (with $C_{s_{1}}^{+}$replaced with $C_{s_{1}}^{-}$) holds for density points of $\{s: \kappa(s)<0\}$.

We are now in a position to derive a contradiction from our assumption that $\lim _{s \rightarrow \sigma_{0}} \theta(w(s))$ does not exist. It follows quite easily from what we have just established, and from Lemma 4.2 and (4.3) that there exist $\eta>0$ and sequences $\left\{s_{k}\right\},\left\{t_{k}\right\}$ tending to $\sigma_{0}$ for which

(i) $s_{k}<t_{k}$,

(ii) the limits of $\theta(z)$ as $z \rightarrow \partial D$ along $C_{s_{k}}^{+}$and $C_{t_{k}}^{-}$exist,

(iii) $\Delta \theta\left(w\left(t_{k}\right) w\left(s_{k}\right)\right) \leq-\eta$.

For each $k$ let $L_{k}$ be the curve $C_{s_{k}} \cup E_{k} \cup C_{t_{k}}$, where $E_{k}$ is the subarc of $C$ from $w\left(s_{k}\right)$ to $w\left(t_{k}\right)$ (with the same orientation as $C$ ). Let $p_{k}$ and $q_{k}$ be the points at which $C_{s_{k}}^{+}$and $C_{t_{k}}^{-}$meet $\partial D$. From (4.5) it follows that $p_{k}, q_{k} \rightarrow 0$. Let $K_{k}$ be the arc of $\partial D$ joining $p_{k}$ and $q_{k}$; obviously, $K_{k} \subset K$ for all sufficiently large $k$.

Let $\alpha_{k}$ and $\beta_{k}$ be the interior angles of the simple closed curve $K_{k} \cup L_{k}$ at $p_{k}$ and $q_{k}$. Note that $K_{k}$ might be $\{p\}$; in this case, $\alpha_{k}$ is the angular size of the sector containing $C_{t_{k}}^{-}$which is bounded by $C_{s_{k}}^{+}$and an arc of $\partial D$, and analogously for $\beta_{k}$. We denote by $\alpha_{k}^{\prime}$ and $\beta_{k}^{\prime}$ the interior angles of the image of this simple closed curve at $f\left(p_{k}\right)$ and $f\left(q_{k}\right)$. Then, by Proposition 2.5 we have

$$
\alpha_{k}^{\prime}+\beta_{k}^{\prime} \geq \frac{m_{j}}{m_{i}}\left(\alpha_{k}+\beta_{k}\right)
$$


We consider that the simple closed curve $K_{k} \cup L_{k}$ is oriented positively, with the orientations of its arcs $K_{k}, C_{s_{k}}^{+}, C_{t_{k}}^{-}$, and $E_{k}$ taken accordingly. Let $\delta_{k}$ denote the change in the tangent direction along $K_{k}$, and let $\delta_{k}^{\prime}$ be the corresponding amount for $f(K)$. Upon taking into account that change in the argument of the tangent along the characteristic arcs $C_{s_{k}}^{+}, E, C_{t_{k}}^{-}$is simply the corresponding change in $\theta$ and the change in the argument of the tangent along their images is the corresponding change in $\phi$, obvious geometric considerations tell us that

$$
\Delta \theta\left(L_{k}\right)=\alpha_{k}+\beta_{k}+\delta_{k}, \quad \Delta \phi\left(L_{k}\right)=\alpha_{k}^{\prime}+\beta_{k}^{\prime}+\delta_{k}^{\prime}
$$

However, by (1.2) it follows that

$$
\Delta \phi\left(C_{s}^{+}\right)=\frac{m_{j}}{m_{i}} \Delta \theta\left(C_{s}^{+}\right), \quad \Delta \phi\left(C_{s}^{-}\right)=\frac{m_{j}}{m_{i}} \Delta \theta\left(C_{s}^{-}\right), \quad \Delta \phi\left(E_{k}\right)=\frac{m_{i}}{m_{j}} \Delta \theta\left(E_{k}\right) .
$$

By (4.8) and the fact that $\Delta \theta\left(E_{k}\right) \leq-\eta$, we have

$$
\begin{aligned}
\frac{m_{j}}{m_{i}}\left(\alpha_{k}+\beta_{k}\right)+\delta_{k}^{\prime} & \leq \alpha_{k}^{\prime}+\beta_{k}^{\prime}+\delta_{k}^{\prime} \\
& =\Delta \phi\left(L_{k}\right) \\
& =\frac{m_{j}}{m_{i}}\left(\Delta \theta\left(C_{t_{k}}^{-}\right)+\Delta \theta\left(C_{s_{k}}^{+}\right)\right)+\frac{m_{i}}{m_{j}} \Delta \theta\left(E_{k}\right) \\
& =\frac{m_{j}}{m_{i}} \Delta \theta\left(L_{k}\right)+\left(\frac{m_{i}}{m_{j}}-\frac{m_{j}}{m_{i}}\right) \Delta \theta\left(E_{k}\right) \\
& \leq \frac{m_{j}}{m_{i}} \Delta \theta\left(L_{k}\right)-\frac{m_{i}^{2}-m_{j}^{2}}{m_{j} m_{i}} \eta \\
& =\frac{m_{j}}{m_{i}}\left(\alpha_{k}+\beta_{k}+\delta_{k}\right)-\frac{m_{i}^{2}-m_{j}^{2}}{m_{j} m_{i}} \eta .
\end{aligned}
$$

Since $\delta_{k}, \delta_{k}^{\prime} \rightarrow 0$ as $k \rightarrow \infty$, this is a clear-cut contradiction, so that $\lim _{s \rightarrow \sigma_{0}} \theta(w(s))$ must indeed exist.

COROLLARY 4.3. Let $D$ and $D^{\prime}$ be Jordan domains and let $f$ be a cps-homeomorphism of $D$ onto $D^{\prime}$ under which open smooth arcs $B$ and $B^{\prime}$ of $\partial D$ and $\partial D^{\prime}$ correspond to each other. Let $C$ and $C^{\prime}$ be $i$ - and $j$-characteristics of $f$, respectively, which exit at $p \in B$. Then, $C$ and $C^{\prime}$ cannot cross in $D$.

Proof. Assume for definiteness that $C$ lies to the left of $C^{\prime}$ (near $p$ ) and that $\mu=m_{j} / m_{i}>1$. Assume that the corollary is false and let $q \in C \cap C^{\prime} \cap D$. Let the arc $p q$ of $C$ be parametrized by $z=z(s), 0 \leq s \leq L$, with $z(0)=p$. Then the $j$-half-characteristic $C^{\prime}(s)$ emanating rightward from $z(s)$ exits at $p$. The simple closed curve $E$ with sides $C(s)=z([0, s])$ and $C^{\prime}(s)$ has interior angles $\beta$ at $p$ and $\pi / 2$ at $z(s)$; the corresponding angles in the image are 
$\gamma$ and $\pi / 2$, where by Proposition $2.5(1 / \mu) \beta \leq \gamma \leq \mu \beta$. Let the changes in $\theta$ along $C(s)$ and $C^{\prime}(s)$ be $\delta$ and $\delta^{\prime}$, when $E$ is traversed in the positive sense. Then $\delta+\delta^{\prime}=\beta+\pi / 2$ and $(1 / \mu) \delta+\mu \delta^{\prime}=\gamma+\pi / 2$. But

$$
\gamma+\frac{\pi}{2}-\frac{1}{\mu} \delta=\mu \delta^{\prime}=\mu\left(\beta+\frac{\pi}{2}-\delta\right) \geq \gamma+\mu \frac{\pi}{2}-\mu \delta
$$

which is impossible since $\delta \rightarrow 0$ as $s \rightarrow 0$.

5. Boundary behavior of $\theta_{f}$ and $\phi_{f}$. In this section we show that if $f$ is a cps-homeomorphism of $D$ onto $D^{\prime}$ under which open $C^{1} \operatorname{arcs} B \subset \partial D$ and $B^{\prime} \subset \partial D^{\prime}$ correspond to each other, then at each point $p \in B$ the functions $\theta$ and $\phi$ are well-behaved, a fact that may be interpreted as indicating the extent to which the cryptocrystalline nature of a solidified lamina persists right up to its boundary. In the context of conformal mappings $g$, there is the theorem of Lindelöf (see [12, Theorem 10.1]), which says that in this situation, the argument of $g^{\prime}$ has a continuous extension to $D \cup B$. We show that there is an analogue of this theorem for cps-mappings, except that the possibility of jump discontinuities in $\theta$ and $\phi$ arises.

We begin with some notational conventions. First of all, when considering the boundary behavior of $f$ at $p \in B$, it constitutes no loss of generality to assume that $\partial D$ is a $C^{1}$ Jordan curve, so that in particular all characteristics have finite length by Proposition 2.9. When discussing a characteristic $C$ which exits $D$ at $p$, we use the arc length parametrization $z=z_{C}:\left[0, \lambda_{1}(C)\right] \rightarrow \bar{D}$, with $z(0)=p$. Here we are dealing with full characteristics, that is, $z\left(\lambda_{1}(C)\right)$ is the other exit point of $C$. We denote the set of full $k$-characteristics exiting at $p$ by $\mathscr{C}_{k}(f, p)$ or simply by $\mathscr{C}_{k}(p)$. Furthermore, $\alpha(C, p) \in[0, \pi]$ will denote the angle formed by the characteristic $C$ and $\partial D$ at $p$, specifically, $\alpha(C, p)=$ $\arccos \left(z_{C}^{\prime}(0) \cdot v\right)$, where $v$ is the positively oriented unit tangent to $\partial D$ at $p$. For a given cps-homeomorphism $f$ of $D$ onto $D^{\prime}$, we denote by $E(f)$ the set of all points of $\partial D$ at which some characteristic of $f$ exits. An $i$-fan at $p$ is an $\mathscr{F}_{\mathcal{C}} \subset \mathscr{C}_{i}(f, p)$ which is maximal with respect to the property that any two characteristics in $\mathscr{F}$ are connected by a $j$-arc.

We define the HP-function $\bar{\theta}(z)=\bar{\theta}\left(z, \alpha_{R}, \alpha_{L}, \delta_{R}, \delta_{L}\right)$ as follows:

$$
\bar{\theta}\left(r e^{i \tau}\right)= \begin{cases}\alpha_{R}, & -\frac{\pi}{2}<\tau<\alpha_{R}, \\ \tau, & \alpha_{R} \leq \tau \leq \alpha_{R}+\delta_{i}, \\ \alpha_{R}+\delta_{R}, & \alpha_{R}+\delta_{R}<\tau<\alpha_{R}+\delta_{R}+\frac{\pi}{2}, \\ \tau-\frac{\pi}{2}, & \alpha_{R}+\delta_{R}+\frac{\pi}{2} \leq \tau \leq \pi-\alpha_{L}, \\ \alpha_{R}+\delta_{R}+\delta_{L}, & \pi-\alpha_{L}<\tau<\frac{3}{2} \pi,\end{cases}
$$


where $\alpha_{R}, \alpha_{L}, \delta_{R}, \delta_{L} \geq 0$,

$$
\begin{gathered}
\alpha_{R}+\alpha_{L}+\delta_{R}+\delta_{L}=\frac{\pi}{2} \\
\tan ^{-1}\left(\frac{m_{j}}{m_{i}} \tan \alpha_{R}\right)+\frac{m_{j}}{m_{i}} \delta_{R}+\frac{m_{i}}{m_{j}} \delta_{L}+\tan ^{-1}\left(\frac{m_{i}}{m_{j}} \tan \alpha_{L}\right)=\frac{\pi}{2}
\end{gathered}
$$

Note that although we are primarily interested in $\theta$ in the half-plane, we have made the first sector $\left(-\pi / 2, \alpha_{R}\right)$, rather than $\left(0, \alpha_{R}\right)$, and similarly for the last sector, in order to accommodate points $p \in \partial D$ at which the tangent line contains points of $D$ arbitrarily close to $p$. Furthermore, we define

$$
\bar{\phi}(z)=\bar{\phi}\left(z, \alpha_{R}, \alpha_{L}, \delta_{R}, \delta_{L}\right)=\bar{\theta}\left(z, \beta_{R}, \beta_{L}, \frac{m_{j}}{m_{i}} \delta_{R}, \frac{m_{i}}{m_{j}} \delta_{L}\right),
$$

where

$$
\beta_{R}=\tan ^{-1}\left(\frac{m_{j}}{m_{i}} \tan \alpha_{R}\right), \quad \beta_{L}=\tan ^{-1}\left(\frac{m_{i}}{m_{j}} \tan \alpha_{L}\right) .
$$

It is easy to see that conditions (5.2) imply that there is a unique $\left(m_{i}, m_{j}\right)$ homeomorphism $h(z)=h\left(z, \alpha_{R}, \alpha_{L}, \delta_{R}, \delta_{L}\right)$ of the upper half-plane onto itself with $h(0)=0, \theta_{h}=\bar{\theta}$, and $\phi_{h}=\bar{\phi}$. We prove the following theorem.

THEOREM 5.1. Let $D$ and $D^{\prime}$ be Jordan domains and let $f$ be a cps-homeomorphism of $D$ onto $D^{\prime}$ under which open smooth arcs $B$ and $B^{\prime}$ of $\partial D$ and $\partial D^{\prime}$ correspond to each other. Then for each point $p \in B$, there exist $\alpha_{R}, \alpha_{L}, \delta_{R}, \delta_{L} \geq 0$ satisfying (5.2) such that for some integers $n$ and $n^{\prime}$,

$$
\begin{array}{r}
\theta_{f}(z)-\xi-\bar{\theta}\left(e^{-i \xi} z, \alpha_{R}, \alpha_{L}, \delta_{R}, \delta_{L}\right) \longrightarrow \frac{n \pi}{2}, \\
\phi_{f}(z)-\xi^{\prime}-\bar{\phi}\left(e^{-i \xi} z, \alpha_{R}, \alpha_{L}, \delta_{R}, \delta_{L}\right) \longrightarrow \frac{n^{\prime} \pi}{2},
\end{array}
$$

as $z \rightarrow p$ in $D$, where $e^{i \xi}$ and $e^{i \xi^{\prime}}$ are the positively oriented unit tangents to $\partial D$ and $\partial D^{\prime}$ at $p$ and $f(p)$, respectively.

Here the correct ordering of $m_{i}$ and $m_{j}$ is such that the fans of $f$ and $h$ "match up"; the integers $n$ and $n^{\prime}$ are necessary to compensate for the normalization implicit in the definitions of $\bar{\theta}$ and $\bar{\phi}$. The proof essentially amounts to showing that $f$ actually has fans matching those of $\bar{\theta}$ at each point of $\partial D$. We accomplish this by first showing that if there is a characteristic $C$ exiting at $p$, then the corresponding fans exist, and that $\theta$ and $\phi$ satisfy (5.5) at $p$; there are, however, some technical complications in showing that these desired 
limits hold as $z \rightarrow p$ to the right (left) of the right (left) fan. Then we show that $E(f)$ is dense in $B$ and finally that $E(f) \cap B$ is closed in $B$, from which it follows that, in fact, $E(f) \supset B$. It is to be noted that $E(f) \supset B$ is not at all obvious since not all HP-nets on smoothly bounded Jordan domains have exiting characteristics at all boundary points. For this reason the proof that $E(f) \cap B$ is closed in $B$ does require a fairly lengthy argument.

We begin with several lemmas.

LEMMA 5.2. Let $p \in B$, let $\epsilon_{n} \rightarrow 0$, let $\left\{Q_{n}\right\}$ be a sequence of nondegenerate curvilinear quadrilaterals with vertices $p, b=b_{n}, c=c_{n}$, and $d=d_{n}$ (in clockwise order), and let $\alpha=\alpha_{n}$ be the interior angle of $Q_{n}$ at $p$. Assume furthermore that

(i) $p d \subset B$,

(ii) $p b$ and $d c$ are $i$-characteristics and $b c$ is a $j$-characteristic,

(iii) $\lambda_{1}(\theta(p b)), \lambda_{1}(\theta(b c))<\epsilon_{n}$,

(iv) $\operatorname{diam}\left(Q_{n}\right)<\epsilon_{n}$,

(v) $\alpha \in[0, \pi / 2]$.

Then $\lambda_{1}\left(\theta\left(Q_{n}\right)\right) \rightarrow 0$.

Proof. In this proof we will in some instances suppress the subscript $n$; this should cause no confusion. Let $C=C_{n}$ be the complete $i$-characteristic of which $p b$ is an initial arc. Without loss of generality we can assume that $\arg z_{C}^{\prime}(s)=\theta\left(z_{C}(s)\right)+\pi / 2$ and that the argument of the positive tangent to $\partial D$ at $p$ is 0 . Let $\bar{\theta}_{n}=\lim _{s \rightarrow 0} \theta\left(z_{C}(s)\right)=\alpha-\pi / 2$. Note that in light of (v), $\bar{\theta}_{n} \in[0,-\pi / 2]$. If the conclusion of the lemma is not valid, then there is some $\xi \in(0,1 / 10)$ such that for arbitrarily large $n$ there is a $w=w_{n} \in Q_{n}$, and a $\xi_{n}$ with $\xi<\left|\xi_{n}\right|<2 \xi$ for which

$$
\begin{gathered}
\theta\left(w_{n}\right)=\bar{\theta}_{n}+\xi_{n}, \\
\frac{\pi}{2}+2 \xi \geq\left|\theta\left(w_{n}\right)\right| \geq \frac{\xi}{2}, \\
\left|\theta\left(w_{n}\right)+\frac{\pi}{2}\right| \geq \frac{\xi}{2}
\end{gathered}
$$

Passing to a subsequence, we can assume that these conditions hold for all $n$. Let $C^{\prime}$ be the maximal $j$-arc passing through $w_{n}$ contained in $Q_{n}$. It is clear that for sufficiently large $n, C^{\prime}$ cannot join a point of $p b$ to one of $c d$ since then it would be the $j$-side of a characteristic quadrilateral whose opposite side is $b c$. By the HP-property, this would imply that $\lambda_{1}\left(\theta\left(C^{\prime}\right)\right)<\epsilon_{n}$ by (iii), and therefore, (also by (iii)) that for all $z \in C^{\prime},|\theta(z)-\bar{\theta}|<2 \epsilon_{n}$, which contradicts (5.6).

We next observe that for all sufficiently large $n, C^{\prime}$ cannot have both of its endpoints on $p d$. To see this, assume that $C^{\prime}$ joins the endpoints of a subarc $E$ of $p d \subset B$ and let $\beta_{1}$ and $\beta_{2}$ be the interior angles of the curvilinear bilateral $C^{\prime} \cup E$. Then in light of Proposition 2.6 and (iv), $\beta_{1}$ and $\beta_{2}$ tend to 0 as $n \rightarrow$ $\infty$, so that for sufficiently large $n$ they are both smaller than the number $\tau_{0}$ 
of Proposition 2.7. If there is some point $u$ of $C^{\prime}$ which is not joined to $b c$ by an $i$-arc in $Q$, then $u$ would have to be joined to $p d$ by an $i$-arc lying in $Q_{n}$ but outside of $C^{\prime} \cup E$. But a simple application of Proposition 2.7 shows that this cannot be. Thus $C^{\prime}$ is the $j$-side of some characteristic quadrilateral whose opposite side is on the $j$-arc $b c$. Then in light of the HP-property and (iii), $\lambda_{1}\left(\theta\left(C^{\prime}\right)\right) \rightarrow 0$. But then, since $\beta_{1}, \beta_{2} \rightarrow 0$ and the tangent to $\partial D$ at $p$ has argument 0 , the fact that $\left|\theta\left(w_{n}\right)\right| \leq \pi / 2+2 \xi$ implies that $\theta\left(w_{n}\right) \rightarrow 0$, which contradicts (5.7).

Thus, for sufficiently large $n$, either

(1) $C^{\prime}$ joins a point $q \in p d$ to a point $t \in p b$, or

(2) $C^{\prime}$ joins a point $q \in p d$ to a point $t \in d c$.

We claim that in either of these cases $\lambda_{1}\left(\theta\left(C^{\prime}\right)\right) \rightarrow 0$ as $n \rightarrow 0$. Let $C^{\prime}$ be parametrized by $\omega(s), 0 \leq s \leq l=l_{n}$, with $\omega(0)=t$. If for all $s, \omega(s)$ is joined to $b c$ by an $i$-arc, then, as above, by the HP-property and (iii), $\lambda_{1}\left(\theta\left(C^{\prime}\right)\right)<\epsilon_{n}$. Thus assume that there is some $s$ for which $\omega(s)$ is joined to $p d$ by the $i$-halfcharacteristic emanating from $C^{\prime}$ leftwards in case (1) and rightwards in case (2), and let $\sigma=\sigma_{n}$ be the smallest such $s$. Then, as before, $\lambda_{1}(\theta(\omega([0, \sigma])))<$ $\epsilon_{n}$. Also, the complete $i$-characteristic through $\omega(\sigma)$ must join two points of $p d$ in $Q_{n}$, and it is clear that the same must be the case for the complete $i$-characteristic through all $\omega(s)$ for $\sigma \leq s \leq l$. Let $F$ be the $i$-characteristic through $\omega(\eta)$, for some $\eta \in[\sigma, l)$, and say that it joins the endpoints $p_{1}$ and $p_{2}$ (in this order when $p d$ is traversed positively) of an arc $E \subset p d$. Let $F$ be parametrized by $\zeta(s), 0 \leq s \leq \rho$ with $\zeta(0)=p_{1}$. By taking into account that by Proposition 2.6 the interior angles $\beta_{1}$ and $\beta_{2}$ of the bilateral $E \cup F$ at $p_{1}$ and $p_{2}$ must tend to 0 as $n \rightarrow \infty$, it follows from Proposition 2.7 that $d \theta(\zeta(s)) / d s$ must be uniformly bounded above by some number $\tau_{1}>0$. From this it easily follows that for $s_{1}<s_{2}$, we must have $\theta\left(\zeta\left(s_{2}\right)\right)<\theta\left(\zeta\left(s_{1}\right)\right)+2 \epsilon_{n} \tau_{1}$, for $n$ sufficiently large. But then the minimum of $\theta\left(\zeta\left(s_{2}\right)\right)-\theta\left(\zeta\left(s_{1}\right)\right)$ must also tend to 0 as $n \rightarrow \infty$, since $\left|\Delta \theta\left(p_{1} p_{2}\right)\right|$ tends to 0 . Thus, we indeed have that

$$
\delta_{n}=\lambda_{1}\left(\theta\left(C^{\prime}\right)\right) \longrightarrow 0, \text { as } n \longrightarrow \infty .
$$

In case (1) we have a contradiction since $\lambda_{1}\left(\theta\left(t w_{n}\right)\right) \rightarrow 0$, together with (iii), implies that $\theta\left(w_{n}\right)-\bar{\theta}_{n} \rightarrow 0$, which is impossible in light of (5.6). In case (2) for sufficiently large $n, \theta\left(w_{n}\right)-\bar{\theta}_{n}=\xi_{n}>0$, since if $\xi_{n}>0, C^{\prime}$ would meet $p b$ because $\lambda_{1}\left(\theta\left(C^{\prime}\right)\right) \rightarrow 0$. Replace $\epsilon_{n}$ by $\max \left\{\epsilon_{n}, \delta_{n}\right\}$. Let $\zeta=\zeta(s), 0 \leq s \leq L$, parametrize $d c$ with $\zeta(0)=d$. Let $t=\zeta\left(s_{0}\right)$. Then $\arg \zeta^{\prime}\left(s_{0}\right)$ is within $\epsilon_{n}$ of $\bar{\theta}+\pi / 2+\xi_{n}$. Since the inclination of $d c$ at $c$ is within $2 \epsilon_{n}$ of $\bar{\theta}+\pi / 2$, there is a subinterval $\left[s_{1}, s_{2}\right]$ of $\left[s_{0}, L\right]$ on which that $\arg \zeta^{\prime}(s)$ varies from $\bar{\theta}+\pi / 2+$ $\xi_{n}-\epsilon_{n}$ to $\bar{\theta}+\pi / 2+2 \epsilon_{n}$. Since by (iv) $\zeta\left(\left[s_{1}, s_{2}\right]\right)$ is contained in $N\left(p b, 2 \epsilon_{n}\right)$, the length of the $\operatorname{arc} \zeta\left(\left[s_{1}, s_{2}\right]\right)$ is certainly less than $10 \epsilon_{n}$. Therefore, there is a point on this arc at which the curvature is least $\left(\xi-3 \epsilon_{n}\right) / 10 \epsilon_{n}$ and at which $d c$ is convex towards the inside of $Q_{n}$. This implies that the full characteristic containing $C^{\prime}$ intersects $\partial D$ at a point $e$ outside of $Q_{n}$ at distance at most 
$3 \epsilon_{n}+10 \epsilon_{n} /\left(\xi-3 \epsilon_{n}\right) \rightarrow 0$ from $p$. However, by Proposition 2.6, the interior angles of the bilateral formed by $C^{\prime}$ and the arc qe of $\partial D$ tend to 0 . But this is inconsistent with the facts that the inclination of the tangent to $C^{\prime}$ at $w_{n}$ is bounded away from 0 and $\delta_{n}=\lambda_{1}\left(\theta\left(C^{\prime}\right)\right) \rightarrow 0$. This is the desired contradiction.

LEMMA 5.3. Let $f$ have an $i$-fan F at $p \in \partial D$. If F has a rightmost (leftmost) characteristic $C$ for which $0<\alpha(C, a) \leq(\pi / 2)(\pi / 2 \leq \alpha(C, p)<\pi)$. Then $\theta(z)$ has a limit as $z \rightarrow a$ in $D$ to the right (left) of $C$.

Proof. For definiteness we deal with the rightmost characteristic case, the leftmost case being treated analogously. For each positive integer $n$ there is a point $b_{n} \in C$ such that $\lambda_{1}\left(p b_{n}\right), \lambda_{1}\left(\theta\left(p b_{n}\right)\right)<1 / n$. Let $C^{\prime}$ be the $j$-halfcharacteristic emanating rightwards from $b_{n}$, parametrized by $\zeta=\zeta(s)$, with $\zeta(0)=b_{n}$. Let $C(s)$ denote the $i$-half-characteristic emanating rightwards from $\zeta(s)$. Then, since $C$ is the rightmost characteristic in $\mathscr{F}_{F}$, there is an $s_{0}>0$ such that $p \notin \overline{C(s)}$ for $s \in\left(0, s_{0}\right]$, and, by making $s_{0}$ smaller if necessary, we can assume that $\lambda_{1}\left(\theta\left(\zeta\left[0, s_{0}\right]\right)\right)<1 / n$. We claim that for all sufficiently small $s$, $C(s) \subset N\left(p b_{n}, 1 / n\right)$. Indeed, were this not so, it would follow from simple continuity properties of the solutions of ordinary differential equations that there is some $\eta>0$ such that for all $\epsilon>0$ there is a $\sigma \in(0, \epsilon)$ for which $C(\sigma)$ has a subarc $E(\sigma) \subset N\left(p b_{n}, 1 / n\right)$ which joins $\zeta(\sigma)$ to a point $q$ within $\epsilon$ of $p$ and another subarc $E^{\prime}(\sigma)$ which joins $q$ to a point $r \in D \backslash N(p, \eta)$. But then it is easy to see that there must be a point $t \in E^{\prime}(\sigma) \cap N(r, 2 \epsilon)$ at which the curvature is greater than $2 / \eta$ and $C(\sigma)$ is concave towards the left side (as one moves away from $\zeta(\sigma)$ ) since otherwise for sufficiently small $\epsilon$, the corresponding $E^{\prime}(\sigma) \cap N(r, 2 \epsilon)$ would be virtually straight lines and would intersect $\partial D$, contradicting the existence of the point $q$. But the $j$-half-characteristic emanating to the left of this $C(\sigma)$ from $t$ would have to intersect $C(\sigma)$ in another point, which is impossible. Let $c_{n}=\zeta(s)$, where $s<s_{0}$ and $C(s) \subset N\left(p b_{n}, 1 / n\right)$, and let $d_{n}$ be the point at which $C(s)$ exits $D$. The desired conclusion now follows from a simple application of Lemma 5.2.

LEMMA 5.4. Let $f$ have an $i$-fan $\mathscr{F}$ at $p \in \partial D$. If $\inf \{\alpha(C, p): C \in \mathscr{F}\}=0$ $(\sup \{\alpha(C, p): C \in \mathscr{F}\}=\pi)$, then for any $\epsilon>0$ there is $a C \in \mathscr{F}$ and $a \delta>0$ such that $\lambda_{1}(\theta(G \cap N(p, \delta)))<\epsilon$, where $G$ is the part of $D$ to the right (left) of $C$.

Proof. It is sufficient to handle the case in which $\inf \{\alpha(C, p): C \in \mathscr{F}\}=0$. Let $C \in \mathscr{F}$ be such that $\alpha(C, p)<\epsilon$. Without loss of generality we can assume that $\arg z_{C}^{\prime}(s)=\theta\left(z_{C}^{\prime}(s)\right)$. Let $C^{\prime}(s)$ denote the $j$-half-characteristic emanating to the right of $z_{C}(s)$ considered as oriented from $z_{C}(s)$ towards $\partial D$. We consider two cases.

CASE 1. For each sufficiently small $\epsilon>0$, there is an $s_{0}=s_{0}(\epsilon)<\epsilon$ such that all points of $C^{\prime}(s)$ are joined to $p$ by an $i$-arc for all $s<s_{0}$. Then it is easy to see that $\lambda_{1}\left(\theta\left(C^{\prime}(s)\right)\right)<\epsilon$, and in fact that for $s<s_{0}, C^{\prime}(s)$ is everywhere concave 
towards $p$ and that the curvature at all points of $C^{\prime}(s)$ is asymptotic to $1 / s$ as $s \rightarrow 0$. From this it follows for sufficiently small $\epsilon$, that $T=\cup\left\{C^{\prime}(s): s<s_{0}\right\}$ contains a set of the form $G \cap N(p, \delta)$ as in the statement. But $\lambda_{1}(\theta(T)) \leq$ $\lambda_{1}\left(\theta\left(z\left(0, s_{0}\right)\right)\right)+\epsilon<2 \epsilon$, for sufficiently small $\epsilon$. Thus the lemma is established in this case.

CASE 2. For arbitrarily small $\epsilon>0$ there are arbitrarily small $s$ such that there are $i$-half-characteristics emanating rightwards from $C^{\prime}(s)$ which do not exit at $p$. In this case for each positive integer $n$, pick a particular such $\epsilon<1 / n$, and a corresponding $s<1 / n$ for which $\lambda_{1}\left(\theta\left(p z_{C}(s)\right)\right)<1 / n$ and such that there are $i$-half-characteristics emanating rightwards from $C^{\prime}(s)$ which do not exit at $p$. Let $b_{n}=z_{C}(s)$. Then, it is easy to see that there is a $c_{n} \in C^{\prime}(s)$ such that the $i$-half-characteristic emanating rightwards from $c_{n}$ exits $D$ at a point $d_{n} \neq p$ and such that $c_{n} d_{n} \subset N(p, 2 / n)$. The desired conclusion now follows easily from Lemma 5.2.

LEMmA 5.5. Let $C, C^{\prime} \in C_{i}(f, p)$ be joined by a j-arc $A$. Then the length of the translate of $A$ down to $q \in C$ tends to 0 as $q \rightarrow p$.

Proof. Let $A$ be parametrized by $w=w(s), 0 \leq s \leq L$, with $w(0) \in C$. Since each point of $A$ is connected to $p$ by an $i$-arc between $C$ and $C^{\prime}$, it is clearly enough to show that the conclusion is valid under the additional assumption that $\lambda_{1}(\theta(A))<1 / 10$. But then, if the length of the translates of $A$ does not tend to 0 , there is a $j$-arc joining $p$ to a point of $C^{\prime}$, which is impossible by Corollary 4.3.

It follows immediately from this lemma and the HP-property that for a fan $\mathscr{F},\{\alpha(C, p): C \in \mathscr{F}\}$ is an interval. The length of this interval, which gives the angular aperture of $\mathscr{F}_{F}$, will be denoted by $\delta(\mathscr{F})$. It also follows from this lemma that if $i$-characteristics $C, C^{\prime}$, and $C^{\prime \prime}$ exit at $p$ and the pairs $C, C^{\prime}$ and $C^{\prime}, C^{\prime \prime}$ are joined by $j$-arcs, then so are $C$ and $C^{\prime \prime}$. The following is also a straightforward consequence of Lemma 5.5 .

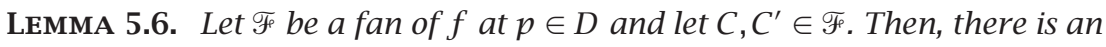
integer $n$ such that $\theta_{f}(z)-\arg (z-p) \rightarrow n \pi / 2$ as $z \rightarrow p$ in the closed subregion of $D$ between $C$ and $C^{\prime}$.

LEMмA 5.7. Let $f$ have an $i$-fan $\mathscr{F}$ at $p \in \partial D$. If the infimum of $\alpha(C, p)>0$ (supremum of $\alpha(C, p)<\pi$ ), then $\mathscr{F}_{\mathrm{F}}$ has a rightmost (leftmost) characteristic.

Proof. We consider the rightmost case. Let $\beta$ be the lower endpoint of the interval $\{\alpha(C, p): C \in \mathscr{F}\}$. Obviously, we can assume that for all $\epsilon>0$ there is a $C^{\prime} \in \mathscr{F}_{F}$ with $\alpha\left(C^{\prime}, p\right)=\beta+\epsilon$ since otherwise the interval would be $\{\beta\}$. Let $C^{\prime \prime}$ be the $j$-half-characteristic emanating rightwards from $z_{C^{\prime}}\left(s_{0}\right)$, where $s_{0}<\epsilon$ is so small that $\lambda_{1}\left(\theta\left(z_{C^{\prime}}\left(0, s_{0}\right)\right)\right)<\epsilon$. Let $w=w(\sigma), 0 \leq \sigma<L$, be the arc length parametrization of $C^{\prime \prime}$, with $w(0)=z_{C^{\prime}}\left(s_{0}\right)$. Let $E(\sigma)$ denote the $i$-characteristic through $w(\sigma)$. If $E(\sigma)$ exits at $p$, then $E\left(\sigma^{\prime}\right)$ exits at $p$ 
for all $\sigma^{\prime} \in[0, \sigma]$ so that for all such $\sigma^{\prime}, E\left(\sigma^{\prime}\right) \in \mathscr{F}$. From this we conclude that if $E(\sigma)$ exits at $p$, then $\lambda_{1}(\theta(w([0, \sigma])))<\epsilon$. But then for $\epsilon$ sufficiently small there is a $\sigma_{0}<L$ for which $E(\sigma)$ does not exit at $p$. Indeed, if this were not so, then as $\epsilon \rightarrow 0$ we would have curvilinear triangles with right angle at $w(0)$, such that along each of the three sides the inclination of the tangent is contained in an interval of length $\epsilon$ (making them virtually straight line segements), and such that the angle at $p$ tends to 0 . However, it is clear that the ratio of the length of the side opposite to $p$ to that of the side $z_{C^{\prime}}\left(\left[0, s_{0}\right]\right)$ is bounded away from 0 , which is obviously inconsistent with these conditions. The desired rightmost characteristic is then the one containing $E\left(\sigma_{1}\right)$, where $\sigma_{1}=\sup \{\sigma: E(\sigma)$ exits at $p\}$.

Proof OF Theorem 5.1. Say we have an $i$-characteristic $C$ exiting at $p$; without loss of generality, we can assume that $p=0$ and that the positively oriented unit tangent vector at $p$ is 1 . We also assume that $0 \leq \alpha(C, p) \leq \pi / 2$, the opposite case being handled analogously. Obviously, there is an $i$-fan $\mathscr{F}_{i}$ at $p$. Let $I_{i}=\left\{\alpha(C, p): C \in \mathscr{F}_{i}\right\}$. By Lemma $5.7, \mathscr{F}_{i}$ will have a rightmost (leftmost) characteristic unless the lower (upper) endpoint $I_{i}$ is $0(\pi)$. In the case that the lower endpoint is 0 , we define $\alpha_{R}=0$; otherwise, we define it to be $\alpha(C, p)$, where $C$ is the rightmost characteristic in $\mathscr{F}_{i}$.

Let $\delta_{R}=\delta\left(\mathscr{F}_{i}\right)$. Then we have $\delta_{R}+\alpha_{R} \leq \pi / 2$. To see this, say $\delta_{R}+\alpha_{R}>\pi / 2$. We denote by $\alpha_{L}$ the angular size of the sector lying to the left of $\mathscr{F}_{i}$. It follows from Lemmas 5.3 and 5.4 that the limit of $\theta(z)$ as $z \rightarrow p$ in each of these sectors exists. Then $\alpha_{R}, \alpha_{L} \leq \pi / 2$ and $\alpha_{L}+\delta_{R}+\alpha_{R}=\pi$. Let $\mu=m_{j} / m_{i}$. It is clear from (1.2), together with Lemma 5.6, that $\delta\left(f\left(\mathscr{F}_{i}\right)\right)=\mu \delta_{R}$. In addition, simple trigonometry, together with Lemma 5.3, implies that the inclination of the image of the rightmost characteristic of $\mathscr{F}_{i}$ is $\tan ^{-1}\left(\mu \tan \alpha_{R}\right)$ and similarly for the image of its leftmost characteristic. It therefore follows that

$$
\tan ^{-1}\left(\mu \tan \alpha_{R}\right)+\tan ^{-1}\left(\mu \tan \alpha_{L}\right)+\mu \delta_{R}=\pi
$$

(Minor modifications show that this equation remains valid in the cases where no rightmost and/or leftmost characteristic exists.) This is clearly impossible since for $\mu<1(\mu>1)$, apart from trivial exceptions, all three terms on the right-hand side of (5.10) are, respectively, less (greater) than $\alpha_{R}, \alpha_{L}, \delta_{R}$. In fact, $\delta_{R}+\alpha_{R}<\pi / 2$, unless we are in the "degenerate case" in which $\alpha_{R}=\pi / 2$ and $\delta_{R}=0$, which we discuss at the end of the next paragraph.

First, we assume that $\delta_{R}+\alpha_{R}<\pi / 2$. In this case there is a $j$-characteristic emanating from $p$ and orthogonal to the leftmost $i$-characteristic in $\mathscr{F}_{i}$. To see that such an orthogonal characteristic exists, let $q$ be a point of the leftmost $i$-characteristic of $\mathscr{F}_{i}$ (near $p$ ) and let $A$ be a (short) $j$-arc emanating from $q$ away from $\mathscr{F}_{i}$. Then the length of the translates of $A$ along this $i$ characteristic towards $p$ are bounded below (and lie in $D$ ), since otherwise this $i$-characteristic would not be the leftmost one in $\mathscr{F}_{i}$. A simple limit argument 
then gives the required $j$-characteristic $J$. It follows from a simple argument based on Corollary 4.3 that no $j$-characteristic to the right of $J$ exits at $p$, so that $J$ is the rightmost characteristic of a $j$-fan $\mathscr{F}_{j}$. Let $\delta_{L}=\delta\left(\mathscr{F}_{j}\right)$ and let $\alpha_{L}$ be the acute angle between $\partial D$ and the leftmost characteristic of $\mathscr{F}_{j}$ if such exists, or 0 otherwise. The limit relationship between $\theta, \phi$ and $\bar{\theta}, \bar{\phi}$ follows from Lemmas 5.3, 5.4, and 5.6, together with simple trigonometry and (1.2). In the degenerate case we have $\alpha_{R}=\pi / 2$ and $\delta_{R}=0$. But then it follows from Lemma 5.3 that the desired limits exist.

Next, we show that $E(f)$ is dense in $B$. Let $p \in B$ and let $\epsilon>0$ be such that $N(p, 2 \epsilon) \cap \partial D \subset B$. If $\theta$ is Lipschitz continuous in $N(p, \epsilon)$, then it is easy to see that there are characteristics exiting at all points of $B \cap N(p, \epsilon)$. On the other hand, if $\theta$ is not Lipschitz continuous in $N(p, \epsilon)$, then one of $\left|D_{1} \theta\right|$ or $\left|D_{2} \theta\right|$ must be unbounded there, so that some characteristic must exit in $N(p, 2 \epsilon) \cap \partial D \subset B$.

Finally, we show that $E(f)$ is closed in $B$. Let $\left\{p_{n}\right\}$ be a sequence of distinct points in $E(f)$ which converge to $p \in B$. It follows from the above construction that there is a $\delta>0$ such that for each $p_{n}$ there is a characteristic $C_{n}$ exiting at $p_{n}$ for which $\delta \leq \alpha\left(C_{n}, p_{n}\right) \leq \pi-\delta$. By passing to a subsequence, we can assume that all of the $C_{n}$ are $i$-characteristics. Similarly, we may assume that the $p_{n}$ tend monotonically to $p$ from one side; for definiteness, say from the right. Let $C_{n}$ be parametrized by $z=z_{n}(s), 0 \leq s \leq \sigma_{n}$, with $z_{n}(0)=p_{n}$. We regard $C_{n}$ as including its end points. In light of Proposition 2.6, the distance between the endpoints of the $C_{n}$ is bounded below by some $\epsilon_{0}>0$. Since $\left\{C_{n}\right\}$ is a family of compact sets, some subsequence, which for convenience we continue to call $\left\{C_{n}\right\}$, converges to a set $S \subset \bar{D}$ with respect to the Hausdorff metric

$$
d(X, Y)=\inf \{\rho: X \subset N(Y, \rho), Y \subset N(X, \rho)\} .
$$

It is easy to see that $S$ is a connected compact set consisting of a union $i$ characteristics (without their endpoints) and a closed subset $E$ of $\partial D$ and that $p \in E$. If $p$ is not an accumulation point of $E$, that is, if $\operatorname{dist}(E \backslash\{p\},\{p\})>$ 0 , then there is an $i$-characteristic of $f$ joining $p$ to $E \backslash\{p\}$, so we are done. Thus we may assume that there is a sequence $\left\{r_{n}\right\}$ of distinct points of $E \backslash\{p\}$ monotonically approaching $p$, and from this we obtain a contradiction. Because the $p_{n}$ lie to the right of $p$, the $C_{n}$ move to the left, so that the points of $E$ (near $p$ ) lie to the left of $p$, and thus the $r_{n}$ lie to the left of $p$.

We now focus on an individual $r_{k}$ for which $\left|r_{k}-p\right|<\epsilon_{0} / 10$ (where $\epsilon_{0}$ is the lower bound for the distance between the endpoints of the $C_{n}$ ) and such that the inclination of the tangent to $\partial D$ along the (short) $\operatorname{arc} r_{k} p$ is less than $1 / 100$. Let $u_{n}=z_{n}\left(s_{n}\right)$ be a point of $C_{n}$ which minimizes distance to $r_{k}$, so that $u_{n} \rightarrow r_{k}$. Note that

$$
N\left(r_{k},\left|u_{n}-r_{k}\right|\right) \text { lies to the left of } C_{n}
$$


since if this were false for some $n_{0}, C_{n_{0}}$ would have to exit $D$ between $r_{k}$ and $p$, and consequently all the $C_{n}$ for $n>n_{0}$ would be contained in the region bounded by $C_{n_{0}}$ and an arc of $\partial D$ joining $p$ to a point between $r_{k}$ and $p$, contradicting the assumption that $r_{k} \in E$. Consider the strip $S_{n}$ of width $\mid r_{k}-$ $p \mid / 5$ whose sides have the normal direction of $\partial D$ at $r_{k}$ and whose centerline passes through $u_{n}$, and let $W_{n}$ be the arc of $C_{n}$ which joins two points of $S_{n}$ and passes through $u_{n}$. For $w \in W_{n}$, let $A_{w}$ denote the $j$-half-characteristic emanating to the right of $C_{n}$. We have

$$
\lambda_{1}\left(A_{w}\right) \geq \frac{\left|r_{k}-p\right|}{10}
$$

for $n$ sufficiently large, since any $A_{w}$ for which this is not true would cross $C_{n}$ twice. Furthermore,

$$
\left|D_{j} \theta(z)\right| \leq \frac{10}{\left|r_{k}-p\right|} \quad \text { for } z \in A_{w} \cap N\left(w, \frac{\left|r_{k}-p\right|}{10}\right)
$$

for all $w \in W_{n}, n \geq n_{1}$, since, otherwise because $\left|r_{k}-p\right|<\epsilon_{0} / 10$, there would be an $i$-characteristic emanating from $A_{w}$ which crosses $C_{n}$, a contradiction. In addition, it follows from (5.13) that

$$
\frac{d \theta\left(z_{n}(s)\right)}{d s} \geq-\frac{10}{\left|r_{k}-p\right|}, \text { for } z_{n}(s) \in W_{n}
$$

for $n$ sufficiently large; that is, for such $n$ the curvature of $C_{n}$ towards the right is uniformly bounded. Now, from (5.15) and the fact that the subarcs of $W_{n}$ on either side of $u_{n}$ have length bounded below by $\left|r_{k}-p\right| / 10$ and do not touch $\partial D$, it follows that for all $\rho>0$, there is an $\epsilon>0$ such that

$$
N\left(u_{n}, \epsilon\right) \cap W_{n} \subset\left\{z:\left|\arg \left(\frac{z-u_{n}}{\xi}\right)\right|<\rho \text { or }\left|\arg \left(\frac{z-u_{n}}{\xi}\right)-\pi\right|<\rho\right\},
$$

for sufficiently large $n$, where $\xi$ is the positive unit tangent to $\partial D$ at $r_{k}$. From (5.14) it follows by compactness that there is a subsequence of $\left\{A_{u_{n}}\right\}$ which converges to a $j$-characteristic $C_{k}^{\prime}$ emanating from $r_{k}$; from (5.16) it follows that $\alpha\left(C_{k}^{\prime}, r_{k}\right)=\pi / 2$. Since all the $C_{n}$ must cross $C_{k}^{\prime}$, it follows that for all $\rho>0$ there is an $\epsilon>0$ such that (5.16) holds with $u_{n}$ replaced by the point of intersection of $C_{k}^{\prime}$ with $W_{n}$. This in turn implies that the image of $\partial D$ is orthogonal to the image of $C_{k}^{\prime}$ at $f\left(r_{k}\right)$.

We can now use our familiar angle change argument on a positively oriented curvilinear triangle made up of the part of $C_{n}$ between $p_{n}$ and $t_{n, k} \in C_{k}^{\prime}$, the $j$-arc $t_{n, k} r_{k}$, and the (short) boundary arc from $r_{k}$ to $p_{n}$. If the changes in the 
tangent inclination on these pieces are $\alpha, \beta, \gamma$, respectively, and the corresponding changes in the images are $\alpha^{\prime}, \beta^{\prime}, \gamma^{\prime}$, and the interior angle at $p_{n}$ is $\tau$ with a corresponding image angle $\tau^{\prime}$, then $\beta, \gamma, \beta^{\prime}$, and $\gamma^{\prime}$ can be made arbitrarily small for $k$ and $n$ sufficiently large. But $\tau \geq \delta>0$ and $\alpha^{\prime}=\left(m_{i} / m_{j}\right) \alpha$, so that we get a contradiction with Proposition 2.5 since

$$
\alpha+\beta+\gamma=\tau, \quad \alpha^{\prime}+\beta^{\prime}+\gamma^{\prime}=\tau^{\prime} .
$$

This finishes the proof of the theorem.

Before ending this section, we mention the following simple consequence of Theorem 5.1 or, more precisely, of its proof.

COROLLARY 5.8. Let $D$ and $D^{\prime}$ be Jordan domains with smooth boundaries, and let $f$ be a cps-homeomorphism of $D$ onto $D^{\prime}$. Then, there is an at most countable set $S \subset \partial D$ such that $\theta_{f}$ and $\phi_{f}$ have continuous extensions to $\bar{D} \backslash S$.

Proof. Let $S$ denote the set of points $p \in \partial D$ at which $\lim _{z \rightarrow p} \theta_{f}(z)$ or $\lim _{z \rightarrow p} \phi_{f}(z)$ fail to exist (as numbers). From the preceding considerations, it follows that for each $p \in S, \delta_{i}(p)+\delta_{j}(p)>0$, where $\delta_{k}(p)$ denotes the angular size of the $k$-fan of $f$ emanating from $p$. Let $\mathscr{F}_{\mathrm{F}}$ be one of these fans with angular size $\delta>0$. Let $\mathscr{F}^{\prime}$ consist of all $C \in \mathscr{F}_{F}$ for which $\alpha(C, p)>\delta / 2$, other than the leftmost and rightmost characteristics of $\mathscr{F}$. The angular size of this subfan $\mathscr{F}^{\prime}$ of $\mathscr{F}$ is at least $\delta / 2$. It follows from Proposition 2.6 that there is a $\gamma=\gamma(\delta)$ such that $\lambda_{1}(C) \geq \gamma$ for all $C \in \mathscr{F}^{\prime}$. For $p \in S$ for which $\delta_{i}(p)>0$, let $\mathscr{F}_{i}^{\prime}(p)$ denote the corresponding subfan and let $E_{i}(p)$ denote the corresponding set of second exit points. Then,

$$
\lambda_{1}\left(E_{i}(p)\right) \geq \delta_{i}(p) \gamma\left(\delta_{i}(p)\right), \quad E_{i}\left(p_{1}\right) \cap E_{i}\left(p_{2}\right) \text { for } p_{1} \neq p_{2} .
$$

From (5.18) it follows that

$$
\sum_{k=1}^{2} \sum_{p \in S} \delta_{k}(p) \gamma\left(\delta_{k}(p)\right) \leq 2 \lambda_{1}(\partial D),
$$

so that $S$ is indeed at most countable.

6. cps-self-mappings of the half-plane. In this section we use Theorem 4.1, together with an appropriate uniqueness argument, to completely determine the class of all $\left(m_{1}, m_{2}\right)$-homeomorphisms $f$ of the upper half-plane $H=\{z$ : $\mathfrak{I} z>0\}$ onto itself. We do this by showing that for all such $f, \theta=\theta_{f}$ has well-defined values on $\mathbb{R}=\partial H$ and that these values are essentially noncharacteristic in the sense that, disregarding simple exceptions, the initial values of $\theta$ (may be taken to) lie entirely in one of the intervals $(0, \pi / 2)$ or $(\pi / 2, \pi)$. This reduction to a well-posed initial value problem is not straightforward since we do not know without the considerations of Section 5 that $\theta$ is even bounded in 
a neighborhood of each point of $\mathbb{R}$. Throughout this section $f$ will denote an $\left(m_{1}, m_{2}\right)$-homeomorphism of $H$ onto itself. We begin with a series of lemmas, the first of which is the key to the succeeding analysis.

LEMMA 6.1. The mapping $f$ does not have any doubly exiting characteristics.

PROof. Assume, to the contrary, that $f$ has a doubly exiting $i$-characteristic $C$. By the remark immediately following the proof of Proposition 2.6, $C$ must have distinct endpoints $a<b$. If $\alpha$ and $\beta$ are the interior angles of the simple closed curve $C \cup[a, b]$ at $a$ and $b$, then it follows from Proposition 2.6 that $\alpha=\beta=0$. For $z \in C$, let $C_{z}^{\prime}$ be the $j$-half-characteristic emanating from $z$ and lying in the exterior of $C \cup[a, b]$. There is a point $w \in C$ at which $C$ is concave towards the exterior of $C \cup[a, b]$, so that $C_{w}^{\prime}$ joins $w$ to a point $u$ of $\partial H$. By Corollary 4.3, $u<a$ or $u>b$. If $u<a$, then for all $z \in a w \subset C$, the same corollary implies that $C_{z}^{\prime}$ joins $z$ to a point of $(-\infty, a)$; in the opposite case for all $z \in b w \subset C, C_{z}^{\prime}$ joins $z$ to a point of $(b, \infty)$. Application of Proposition 2.7 then gives a contradiction for $z$ sufficiently close to $a$ in the first case or to $b$ in the second case.

LEMMA 6.2. Let $C$ be a characteristic arc of $f$ given by $z=z(s), 0<s<L$. Then, $\kappa(s)=d \arg z^{\prime}(s) / d$ s has a single sign on $(0, L)$; that is, $\kappa(s) \geq 0$ a.e. on $(0, L)$ or $\kappa(s) \leq 0$ a.e. on $(0, L)$.

Proof. If the conclusion were not true, then by Proposition 2.1 there would be $s^{+}, s^{-} \in(0, L)$ such that the orthogonal half-characteristics $C^{+}$issuing from $p^{+}=z\left(s^{+}\right)$to the left of $C$ and $C^{-}$issuing from $p^{-}=z\left(s^{-}\right)$to the right of $C$ join $p^{+}$and $p^{-}$to points $a^{+}$and $a^{-}$of $\mathbb{R}$. We assume that $s^{+}<s^{-}$, the opposite case being handled similarly. Let $C^{\prime}$ denote the closed subarc $p^{+} p^{-}$ of $C$. Let $D$ denote the interior of the simple closed piecewise smooth curve $\left[a^{+}, a^{-}\right] \cup C^{+} \cup C^{-} \cup C^{\prime}$. If the right-hand side of $C$ faces the interior of $D$, then by Proposition 2.8 the half-characteristic $E$ orthogonal to $C$ issuing to the right of $p^{+}$intersects $\partial D$. Since $E \cap C=\left\{p^{+}\right\}$and $E$ cannot touch the interior of either $C^{+}$or $C^{-}, E$ must meet $\left[a^{+}, a^{-}\right] \subset \partial D$. But then $E \cup C^{+}$is a doubly exiting characteristic, which is impossible by the preceding lemma. If $D$ is on the left-hand side of $C$, then an analogous argument produces a doubly exiting characteristic passing through $p^{-}$.

LEMMA 6.3. Any nonexiting characteristic $C$ must be a horizontal straight line.

Proof. Clearly, if $C$ is a nonexiting straight-line characteristic, it must be horizontal. Thus, it is sufficient to show that the curvature is identically 0 on any nonexiting characteristic. Assume, to the contrary, that this is not the case for some such $i$-characteristic $C$ given by $z=z(s),-\infty<s<\infty$. Since by the preceding lemma $\arg z^{\prime}(s)$ is monotone on $(-\infty, \infty)$, there are two cases:

(i) $\arg z^{\prime}(s)$ is unbounded in at least one direction,

(ii) $\arg z^{\prime}(s)$ is bounded in both directions. 
In case (i) it is easy to see that at one end or the other, $C$ must have an infinite inward spiral, which is clearly impossible. In case (ii) $C$ is a U-shaped curve each end of which must extend upward or at worst be horizontal. Thus there must be some point $p$ on $C$ with nonvanishing curvature at which $C$ is concave towards the inside of the $U$. But then by Proposition 2.1, the $j$-half-characteristic $C^{\prime}$ emanating from $p$ into the $\mathrm{U}$ must join $p$ to $\partial H$. But this implies that $C^{\prime}$ intersects $C$ in two points, which is impossible.

LEMMA 6.4. If $f$ has a nonexiting characteristic, then $f$ must be affine with horizontal and vertical characteristics.

Proof. If $f$ has a nonexiting $i$-characteristic $C$, then by the preceding lemma it must be a horizontal straight line. Let $C^{\prime}$ be any orthogonal characteristic. If the tangent to $C^{\prime}$ were nonvertical at any point $p \in C^{\prime}$, then the $i$-characteristic through $p$ would be a straight line intersecting $C$, an obvious contradiction. Thus, $C^{\prime}$ is a vertical line, so that the two families of characteristics are given by $\mathfrak{R} z=$ const and $\mathfrak{I} z=$ const, from which the desired conclusion follows immediately.

Since this lemma gives a complete description of all $f$ possessing a nonexiting characteristic, for the remainder of this section, unless otherwise stated, we assume that all characteristics exit, and so, in light of Lemma 6.1, we assume that they exit exactly once. Each characteristic $C$ will be parametrized by $z=Z_{C}(s), 0 \leq s<\infty$, so that $\alpha_{C}=\arg Z_{C}^{\prime}(0)$ is the angle formed by $C$ with the part of $\mathbb{R}$ to the right of $C$. We adopt the convention $0 \leq \alpha_{C} \leq \pi$. Furthermore, each characteristic splits $H$ into two pieces. Since characteristics belonging to the same family never cross in $H$, it makes sense to speak of a characteristic as lying to the right or left of one belonging to the same family. Obviously, $C_{1}$ lies to the left of $C_{2}$ if and only if $Z_{C_{1}}(0) \leq Z_{C_{2}}(0)$. Since by Lemma 6.2, $\arg Z_{C}^{\prime}(0)$ is monotonic, it follows that

$$
\alpha_{C} \leq \arg Z_{C}^{\prime} \leq \pi \text { for all } s \in[0, \infty)
$$

or

$$
0 \leq \arg Z_{C}^{\prime} \leq \alpha_{C} \text { for all } s \in[0, \infty)
$$

Were this is not so, we would have a doubly exiting characteristic, which is impossible by Lemma 6.1, or a characteristic with an infinite inward spiral, which is also impossible.

LEMMA 6.5. If $C$ is a characteristic of $f$, then all of the orthogonal characteristics must exit in the same component of $H \backslash C$. In fact, this component is the right-hand one if $\arg Z_{C}^{\prime}(s) \in[0, \pi / 2)$ for some $s \geq 0$ and the left-hand one if $\arg Z_{C}^{\prime}(s) \in(\pi / 2, \pi]$ for some $s \geq 0$. 
Proof. Let $C$ be an $i$-characteristic. The first sentence follows easily from Lemma 6.1, as in the proof of Lemma 6.2. For the second conclusion, assume, for example, that $\arg Z_{C}^{\prime}(s) \in[0, \pi / 2)$ for some $s \geq 0$. Let $C^{\prime}$ be the $j$-halfcharacteristic emanating to the right of $Z_{C}^{\prime}(s)$. If $C^{\prime}$ does not meet $\partial H$, then simple geometric considerations imply that as we move along $C^{\prime}$ away from $Z_{C}^{\prime}(s)$, there will be some point $p$ at which $C^{\prime}$ is concave to the left. But then the $i$-half-characteristic emanating leftward from $p$ must meet $\partial H$ but at the same time is trapped inside the infinite subregion of $H$ bounded by $\left\{Z_{C}^{\prime}(\sigma)\right.$ : $\sigma>s\} \cup C^{\prime}$, which is a contradiction. Therefore, $C^{\prime}$ must meet $\partial H$.

If $\alpha_{C}=0$, then because of the monotonicity of $\arg Z_{C}^{\prime}$, this function would have to be nondecreasing and not constantly 0 on $[0, \infty)$. That is, $C$ would have to be concave to the left. But then Lemma 6.5 and Proposition 2.1 would together imply that some orthogonal characteristic exits on both sides of $C$, in contradiction to Lemma 6.1. We obtain a similar contradiction if $\alpha_{C}=\pi$. Thus,

$$
0<\alpha_{C}<\pi \text { for all } C \text {. }
$$

We denote by $\mathscr{F}_{i}$ family $i$-characteristics of $f$.

LEMMA 6.6. Either for all $C \in \mathscr{F}_{i}$ all orthogonal characteristics exit to the left or for all $C \in \mathscr{F}_{i}$ they exit to the right.

Proof. If not, then we would have $C_{1}$ and $C_{2}$ in $\mathscr{F}_{i}$ with orthogonal characteristics exiting to the left and right, respectively. If $Z_{C_{1}}(0) \leq Z_{C_{2}}(0)$, then let $C_{1}^{\prime}$ and $C_{2}^{\prime}$ be infinite $j$-half-characteristics emanating from $C_{1}$ and $C_{2}$ with initial inclinations in $[0, \pi / 2)$ and $(\pi / 2, \pi]$, respectively. The only possibility is that the inclinations are 0 and $\pi$ since otherwise they would cross. But then they must be horizontal lines since otherwise we would have doubly exiting characteristics. Appropriate downward translates of these lines will then coincide, which again produces a doubly exiting characteristic. Thus $Z_{C_{1}}(0)>Z_{C_{2}}(0)$. In this case the only possibility is that $C_{1}$ and $C_{2}$ are vertical lines, since otherwise they would cross. Let $p \in C_{1}$. If the $j$-arc $C$ through $p$ crossed $C_{2}$, then it would exit both to the left and to the right of $C_{2}$, making it a doubly exiting characteristic. Therefore, it must exit in $\left[Z_{C_{2}}(0), Z_{C_{1}}(0)\right)$. But then if we translate the part of $C_{1}$ above $p$ downward along $C$, we will obtain $i$-arcs which are rays with inclination in $(\pi / 2, \pi)$, which will necessarily cross $C_{2}$. This is impossible since distinct $i$-characteristics cannot cross in $H$.

LEMMA 6.7. Let $C_{1}, C_{2} \in \mathscr{F}_{i}$ with $Z_{C_{1}}(0)<Z_{C_{2}}(0)$. Then $\alpha_{C_{1}} \geq \alpha_{C_{2}}$.

Proof. We prove this for the case in which for all $C \in \mathscr{F}_{i}$, the $j$-characteristics crossing it exit to its right, that is, in which all of the characteristics in $\mathscr{F}_{i}$ are concave to the right; the opposite case is handled analogously. If $C_{2}$ is a vertical line, then $C_{1}$ must be one too since otherwise $C_{1}$ would intersect $C_{2}$, so that $\alpha_{C_{1}}=\alpha_{C_{2}}=\pi / 2$. We therefore assume that $C_{2}$ is not a vertical line. It 
follows from the preceding lemma that the characteristics in $\mathscr{F}_{j}$ are concave to the left. By assumption the $j$-half-characteristic $E^{\prime}$ emanating to the left of $C_{2}$ from $Z_{C_{2}}(s)$ is infinite and must therefore intersect $C_{1}$ at some point $Z_{C_{1}}\left(s^{\prime}\right)$. Since $E^{\prime}$ is concave to the left, we have $\alpha_{C_{1}} \geq \arg Z_{C_{1}}^{\prime}\left(s^{\prime}\right) \geq \arg Z_{C_{2}}^{\prime}(s)$. The desired conclusion now follows since $\arg Z_{C_{2}}^{\prime}(s) \rightarrow \alpha_{C_{2}}$ as $s \rightarrow 0$.

LEMMA 6.8. Let $C_{0} \in \mathscr{F}_{i}$ and assume that all $j$-characteristics which cross $C_{0}$ exit $H$ to the right of $C_{0}$. If $C_{0}$ exits $H$ at $x_{0}$ and $x<x_{0}$, then there is an $i$-characteristic of $f$ which exits $H$ at $x$.

Proof. First of all, it is easy to see by a simple compactness argument that the set $E$ of exit points $x \leq x_{0}$ of $C \in \mathscr{F}_{i}$ is closed in $\mathbb{R}$. If $E \neq\left(-\infty, x_{0}\right]$, then there is some $x_{1} \leq x_{0}$ in $E$, such that $\left(x_{2}, x_{1}\right) \cap E=\varnothing$, for some $x_{2}<x_{1}$. Let $C$ be the leftmost $i$-characteristic exiting at $x_{1}$. Let the $j$-half-characteristic $C_{s}^{\prime}$ emanating leftward from $Z_{C}(s)$ be parametrized by $w_{s}(\sigma), \sigma \geq 0$. Then it is easy to see that the $i$-characteristic through $w_{s}(\sigma)$ will exit $H$ at a point $x \neq x_{1}$ arbitrarily near $x_{1}$ for $s, \sigma>0$ sufficiently small, which contradicts the definition of $x_{2}$.

We are now in a position to obtain a description of the initial values of $\theta=\theta_{f}$ and $\phi=\phi_{f}$. It follow from Lemma 6.6 that with appropriate choice of $i$ and $j$, all $j$-characteristics passing through a point of any $i$-characteristic $C$ exit to the right of $C$, and oppositely when $i$ and $j$ are reversed. Thus from Lemma 6.5, we conclude that

$$
\alpha_{C} \in\left(0, \frac{\pi}{2}\right] \text { for all } C \in \mathscr{F}_{i}, \quad \alpha_{C} \in\left[\frac{\pi}{2}, \pi\right) \text { for all } C \in \mathscr{F}_{j} .
$$

Obviously,

all $C \in \mathscr{F}_{i}\left(C \in \mathscr{F}_{j}\right)$ are concave to the right (left).

For $C \in \mathscr{F}_{i}$ we work with the angles $\alpha_{C}$ and call them $\theta^{*}(x)$, where $x \in \partial H$ is the point where the $C$ exits. If we define $\theta^{*}(x)$ to be 0 for all $x$ for which there is no $C \in \mathscr{F}_{i}$ exiting at $x$, then Lemmas 6.7 and 6.8 imply that $\theta^{*}$ is a nonincreasing function on all of $\mathbb{R}=\partial H$; at the jump points of $\theta^{*}$ there are fans of exiting characteristics as described in Section 5. For definiteness we take $\theta^{*}$ to be continuous from the right. From the considerations of Section 5 it follows that there is at least one exiting characteristic at each point of $\partial H$, so that in light of the concavity of the characteristics and the monotonicity of $\theta^{*}$, there are three intervals $I_{\pi / 2}, I, I_{0}$ on which the function $\theta^{*}(x)$ takes only the value $\pi / 2$, only values in $(0, \pi / 2)$, and the value 0 , respectively. Any of these three intervals may be empty; in fact, the only thing that cannot happen is that $\partial H=I_{\pi / 2}$ or $\partial H=I_{0}$ since in the former (latter) case it can easily be seen that all $j$-characteristics ( $i$-characteristics) would be horizontal. Finally, 
if $f$ is of the form $m_{i} x+m_{j} y+x_{0}$ or $m_{j} x+m_{i} y+x_{0}$, then we regard $\theta^{*}$ as being identically 0 or $\pi / 2$, respectively, on all of $\partial H$.

THEOREM 6.9. If $f$ is a cps-homeomorphism of $H$ onto itself, then the function $\theta^{*}=\theta_{f}^{*}$ on $\partial H=\mathbb{R}$ defined in the preceding paragraph (i) is nonincreasing, (ii) is continuous from the right, and (iii) has all of its values in $[0, \pi / 2]$. Furthermore, given any such $\theta^{*}$ and any $m_{1}, m_{2}$, there is a unique $\left(m_{1}, m_{2}\right)$-mapping $f$ for which $\theta_{f}^{*}=\theta^{*}$ and $f(0)=0$.

Comment. This theorem says that the class $\mathscr{A}$ of cps-homeomorphisms $f$ of $H$ onto itself with principal strains $m_{1}$ and $m_{2}$ and for which $f(0)=0$ can be decomposed as $\mathscr{A}=\mathscr{A}_{1} \cup \mathscr{A}_{2}$, where $\mathscr{A}_{1} \cap \mathscr{A}_{2}$ consists of the two linear mappings $m_{1} x+m_{2} y$ and $m_{2} x+m_{1} y$, and where there is a one-to-one correspondence between each $\mathscr{A}_{i}$ and the set of functions satisfying conditions (i), (ii), and (iii) of the statement of the theorem. Here $\mathscr{A}_{i}$ consists of the mappings for which the characteristics corresponding to stretch factor $m_{i}$ are concave to the right.

To finish the proof of Theorem 6.9, we need to show existence and uniqueness of the $f$ corresponding to a given $\theta^{*}$. To establish existence, we initially let $\theta^{*}$ be a strictly decreasing $C^{\infty}$-function with values in some compact subinterval $I$ of $(0, \pi / 2)$ and let

$$
\phi^{*}(x)=\arctan \left(\frac{m_{2}}{m_{1}} \tan \theta^{*}(x)\right) .
$$

By well-known existence theorems for hyperbolic systems, there exists a $C^{\infty}$ solution $\theta, \phi$ of (1.2) in some neighborhood of $\mathbb{R}$ with initial data $\theta^{*}, \phi^{*}$. Now, for such $\theta, \phi$,

$$
\begin{gathered}
\theta_{x}=(\cos \theta) D_{1} \theta-(\sin \theta) D_{2} \theta \\
\phi_{x}=(\cos \theta) D_{1} \phi-(\sin \theta) D_{2} \phi=\frac{m_{1}}{m_{2}}(\cos \theta) D_{1} \theta-\frac{m_{2}}{m_{1}}(\sin \theta) D_{2} \theta .
\end{gathered}
$$

From (6.6), it follows that on $\mathbb{R}=\partial D$ there hold

$$
\begin{aligned}
& D_{1} \theta=\frac{\theta_{x} m_{2}^{2} \sin ^{2} \theta}{\left(m_{1}^{2} \cos ^{2} \theta+m_{2}^{2} \sin ^{2} \theta\right) \cos \theta}<0, \\
& D_{2} \theta=-\frac{\theta_{x} m_{1}^{2} \cos ^{2} \theta}{\left(m_{1}^{2} \cos ^{2} \theta+m_{2}^{2} \sin ^{2} \theta\right) \sin \theta}>0,
\end{aligned}
$$

so that the 1-characteristics are initially concave to the right and the 2-characteristics are initially concave to the left. But then as we move upward away from $\partial H$ along the characteristics, the solution has decreasing $\left|D_{1} \theta\right|$ and $\left|D_{2} \theta\right|$, and furthermore from the concavity of the characteristics, it follows that we always have $\theta(z)$ in $I$. Thus, we can extend the solution little by little into all of $H$ without any singularities developing. By the assignment (6.6) of $\phi^{*}$ the mapping $f$ for which $f(0)=0, \theta_{f}=\theta$ and $\phi_{f}=\phi$ is an $\left(m_{1}, m_{2}\right)$-homeomorphism of $H$ onto itself. 
Now let $\theta^{*}$ be any nonincreasing function on $\mathbb{R}$ with values in $[0, \pi / 2]$ which is continuous from the right, and let $\left\{\theta_{n}^{*}\right\}$ be a sequence of strictly decreasing $C^{\infty}$-functions such that $\overline{\theta_{n}^{*}(\mathbb{R})} \subset(0, \pi / 2)$, and such that for each $a<b$,

$$
\int_{a}^{b}\left|\theta_{n}^{*}(x)-\theta^{*}(x)\right| d x \rightarrow 0 \quad \text { as } n \longrightarrow \infty .
$$

Let $\left(\theta_{n}, \phi_{n}\right)$ be the solution in $H$ of the initial value problem for (1.2) with initial data $\theta_{n}^{*}(x)$ and $\phi_{n}^{*}(x)=\arctan \left(\left(m_{2} / m_{1}\right) \tan \theta_{n}^{*}(x)\right)$, and let $f_{n}$ be the corresponding mapping of $H$ onto $H$ with $f_{n}(0)=0$. By the compactness principle, some subsequence of $\left\{f_{n}\right\}$, which for convenience we continue to call $\left\{f_{n}\right\}$, converges locally uniformly on $\bar{H}$ to an $\left(m_{1}, m_{2}\right)$-homeomorphism $f$ of $H$ onto itself. Since

$$
\frac{\partial f_{n}(x, 0)}{\partial x}=\left(m_{1}^{2} \cos ^{2} \theta_{n}^{*}(x)+m_{2}^{2} \sin ^{2} \theta_{n}^{*}(x)\right)^{1 / 2},
$$

it follows from (6.9) that

$$
\begin{aligned}
f(b)-f(a) & =\lim _{n \rightarrow \infty} f_{n}(b)-f_{n}(a) \\
& =\lim _{n \rightarrow \infty} \int_{a}^{b}\left(m_{1}^{2} \cos ^{2} \theta_{n}^{*}(x)+m_{2}^{2} \sin ^{2} \theta_{n}^{*}(x)\right)^{1 / 2} d x \\
& =\int_{a}^{b}\left(m_{1}^{2} \cos ^{2} \theta^{*}(x)+m_{2}^{2} \sin ^{2} \theta^{*}(x)\right)^{1 / 2} d x,
\end{aligned}
$$

so that $\partial f(x, 0) / \partial x=\left(m_{1}^{2} \cos ^{2} \theta^{*}(x)+m_{2}^{2} \sin ^{2} \theta^{*}(x)\right)^{1 / 2}$ a.e. on $\mathbb{R}$. Because $\theta^{*}$ is continuous from the right, it therefore follows that $\theta_{f}^{*}(x)=\theta^{*}(x)$ for all $x \in \mathbb{R}$, as desired.

Finally, we have to show that given any such function $\theta^{*}$ on $\mathbb{R}$, together with its companion function $\phi^{*}(x)=\arctan \left(\left(m_{2} / m_{1}\right) \tan \theta^{*}(x)\right)$, the corresponding $\left(m_{1}, m_{2}\right)$-self-homeomorphism $f$ of $H$ with $f(0)=0$ is unique; in other words, that the functions $\theta$ and $\phi$ in $H$ corresponding to these boundary functions are uniquely determined in $H$. This is not completely obvious since $\theta^{*}$ and $\phi^{*}$ may not be Lipschitz continuous (nor even continuous for that matter). We deduce uniqueness by "spreading out," in effect, the initial line so as to transform the given problem into a standard Cauchy problem with Lipschitz data.

We begin by proving uniqueness (in the domain of dependence of $[a, b]$ ) under the assumption that $\theta^{*}([a, b]) \subset(\epsilon, \pi / 2-\epsilon)$ for some $\epsilon>0$, that $\theta^{*}$ is nonincreasing, and that it is continuous at $a$ and $b$. The general case follows easily from this, as we indicate below.

Let $\theta(a)=\alpha \geq \beta=\theta(b)$. Without loss of generality, we can assume that $a=0$. Let $\bar{\theta}(x)=\alpha-\theta^{*}(x)$, so that $\bar{\theta}$ is nondecreasing on $[0, b]$ with values in $[0, \alpha-\beta]$. Let $P$ denote the countable set of points of $[0, b]$ at which this function has jumps, the jump at $p$ being denoted by $\delta(p)=\delta_{1}(p)+\delta_{2}(p)$, where $\delta_{1}(p)$ and $\delta_{2}(p)$ are the angles of the associated fans of 1- and 2-characteristics 
emanating from $p$ (see Section 5). For each $n \geq 1$, let $0=a_{0}<a_{1}<\cdots<a_{n}=b$ be a set of points of $[0, \alpha-\beta] \backslash P$ such that $\left|a_{k}-a_{k-1}-1 / n\right|<1 / n^{2}$ for $1 \leq k \leq$ $n$. We define $\xi_{n}\left(a_{k}\right)=a_{k}+\bar{\theta}\left(a_{k}\right), 0 \leq k \leq n$, and let $\xi_{n}$ be linear on each of the intervals $\left[a_{k-1}, a_{k}\right]$. Then obviously, the inverse $\eta_{n}$ of $\xi_{n}$ on the interval $J=[0, b+\alpha-\beta]$ satisfies a Lipschitz condition with constant 1 . Also, if we define $\bar{\theta}_{n}$ on $J$ to be the piecewise linear function for which $\bar{\theta}_{n}\left(\xi_{n}\left(a_{k}\right)\right)=\bar{\theta}\left(a_{k}\right)$, then $\bar{\theta}_{n}$ is also Lipschitz continuous with constant 1 on $J$. Using the compactness of the families $\left\{\eta_{n}\right\}$ and $\left\{\bar{\theta}_{n}\right\}$, we obtain functions $\eta, \bar{\theta}^{*}$ satisfying a Lipschitz condition with constant 1 such that $\eta$ maps $J$ onto $[0, b]$ and $\bar{\theta}^{*}$ maps $J$ onto $[0, \alpha-\beta]$ with derivative 1 on the subintervals of $J$ corresponding to the jumps in $\bar{\theta}$ and such that for each $x$ for which the original $\theta$ is continuous at $\eta(x)$ we have $\bar{\theta}^{*}(x)=\bar{\theta}(\eta(x))$. For $p \in P$ we denote $\lim _{x \rightarrow p^{-}} \theta^{*}(x)$ by $\theta^{-}(p)$ and similarly for $\phi^{*}$. Note that since $\theta^{*}$ and $\phi^{*}$ are continuous from the right, $\theta^{*}(p)$ and $\phi^{*}(p)$ are equal to their limits as $x \rightarrow p^{+}$. Also, by the relationship between the fan angles of $\theta^{*}$ at $p$ and the sizes of their images under $f$ at $f(p)$ established in Section 5 , it follows that

$$
\phi^{-}(p)=\lim _{x \rightarrow p^{-}} \phi^{*}(x)=\phi^{+}(p)+\left(\frac{m_{1}}{m_{2}}\right) \delta_{2}(p)+\left(\frac{m_{2}}{m_{1}}\right) \delta_{1}(p) .
$$

For any $x \in J$ for which $\theta^{*}$, and consequently $\phi^{*}$ also, is continuous at $\eta(x)$, we let $\Theta(x)=\theta^{*}(\eta(x))$ and $\Phi(x)=\phi^{*}(\eta(x))$. On the other hand, on the interval $I \subset J$ corresponding to $p \in P$, we let $\Theta$ vary linearly from $\theta^{-}(p)$ to $\theta^{*}(p)$ and $\Phi$ vary linearly from $\phi^{-}(p)$ to $\phi^{*}(p)$. The functions $\Theta$ and $\Phi$ so defined on the interval $J$ are clearly Lipschitz continuous.

For $t \in J$, let $L_{1}(t), L_{2}(t)$ be the halves of the lines $x-y=t$ and $x+y=t$ emanating upwards from the $x$-axis. We extend $\Theta$ and $\Phi$ to the triangle $T$ bounded by $L_{1}(0)$ and $L_{2}(b+\alpha-\beta)$ by letting $R_{i}^{\prime}=m_{i} \Theta-m_{j} \Phi$ be constant on the each line $L_{i}(\rho)$ for all $\rho \in J$. The functions $\Theta$ and $\Phi$ are obviously Lipschitz continuous on $T$. Now consider a Lipschitz continuous mapping $z=u+i v$ : $T \rightarrow \mathbb{C}$ which satisfies the initial condition

$$
z(x, 0)=\eta(x)
$$

and the differential equations

$$
\arg \left\{z_{x}+z_{y}\right\}=\Theta(x, y), \quad \arg \left\{z_{y}-z_{x}\right\}=\Theta(x, y)+\frac{\pi}{2},
$$

that is,

$$
\begin{gathered}
\left(v_{y}+v_{x}\right) \cos \Theta-\left(u_{y}+u_{x}\right) \sin \Theta=0 \\
\left(v_{y}-v_{x}\right) \sin \Theta+\left(u_{y}-u_{x}\right) \cos \Theta=0
\end{gathered}
$$

Writing these differential equations in the form

$$
V_{y}+V_{x}=-\left(\Theta_{y}+\Theta_{x}\right) U, \quad U_{y}-U_{x}=-\left(\Theta_{y}-\Theta_{x}\right) V,
$$


where $U=u \cos \Theta+v \sin \Theta$ and $V=v \cos \Theta-u \sin \Theta$, and converting them to integral equations, it is easy to see that the linear hyperbolic system (6.15) with initial condition (6.13) has at most one Lipschitz continuous solution. We now consider the restriction to the domain of dependence $D$ corresponding to $[a, b]$ of any $\left(m_{1}, m_{2}\right)$-mapping $f$ corresponding to initial values $\theta^{*}$ and $\phi^{*}$, and define $z: T \rightarrow D$ as follows. For $p \in P$, let $I(p) \subset J$ be the corresponding closed interval. If $t \in I(p)$, let $R(t)$ be the union of the two legs of the closed isosceles right triangle $T(p)$ with base $I(p)$, and for all other $t \in J$ let $R(t)=t$. Note that each $L_{i}(\rho)$ meets a unique $R(t)$. If $\zeta \in T(p)$ for some $p$, then we define $z(\zeta)=$ $p$. If $\{\zeta\}=L_{1}\left(\rho_{1}\right) \cap L_{2}\left(\rho_{2}\right)$ and $\zeta$ is not in any $T(p)$, then we define $\zeta_{i}$, $t_{i}$, by $\left\{\zeta_{i}\right\}=L_{i}\left(\rho_{i}\right) \cap R\left(t_{i}\right)$. We then define $z(\zeta)$ to be the point on the intersection of the 1-characteristic of $f$ through $\eta\left(\rho_{1}\right)$, whose initial inclination is $\Theta\left(\zeta_{1}\right)$, with the 2-characteristic through $\eta\left(\rho_{2}\right)$, whose initial inclination is $\Theta\left(\zeta_{2}\right)+\pi / 2$. This mapping is well defined. Indeed, for each $\rho$ not in any $I(p), p \in P$, the indicated characteristics emanate from $\eta(\rho)$, in light of what we deduced about the fans in Section 5. On the other hand, if $\rho \in I(p)$ for some $p \in P$, then on the left side of any $R(\rho)$, $\Theta$ decreases linearly from $\theta^{-}(p)-\delta_{2}(p)$ to $\theta^{*}(p)$ and $\Phi$ decreases linearly from $\phi^{-}(p)-\left(m_{1} / m_{2}\right) \delta_{2}(p)$ to $\phi^{*}(p)$. Since an analogous statement holds for the right side of $R(\rho)$, we again have that the indicated characteristics are well defined. Obviously, $z(t)=\eta(t)$ for $t \in J$. Because of the definition of $\Theta$ and $\Phi$ in terms of the invariance of $R_{i}^{\prime}=m_{i} \Theta-m_{j} \Phi$ on the $L_{i}$, and the fact that the $R_{i}=m_{i} \theta-m_{j} \phi$ are invariant on the $i$-characteristics of $f$, we see that the inclination of the curve $z\left(L_{1}(\rho)\right)$ at $z(\zeta)$ is $\Theta(\zeta)$ and that that of $z\left(L_{2}(\rho)\right)$ is $\Theta(\zeta)+\pi / 2$, that is, that $\theta(z(\zeta))=\Theta(\zeta)$. In addition, this means that changes in $\Theta(\zeta)$ and $\theta(z(\zeta))$ coincide on segments of the $L_{i}(t)$. It is clear that there is some $K=K\left(m_{1}, m_{2}\right)$, such that $\Theta$ is Lipschitz continuous with constant $K$ on each $L_{i}(t)$. It is also clear that all the characteristics of $f$ in the domain of dependence $D$ have length bounded by some $M<\infty$. Moreover, the initial points of $z\left(L_{j}\left(\rho_{1}\right)\right)$ and $z\left(L_{j}\left(\rho_{2}\right)\right)$ are at most $\left|\eta\left(\rho_{1}\right)-\eta\left(\rho_{2}\right)\right| \leq\left|\rho_{1}-\rho_{2}\right|$. From these last two facts, together with the convexity of the characteristics of $f$ and the fact that $\Delta \Theta$ and $\Delta \theta \circ z$ coincide on segments of the $L_{i}$, it is easy to show using the HP-property that if $S$ is a segment of $L_{i}(\rho)$, then the length of $z(S)$ is at most $(K M+1) \lambda_{1}(S)$, so that $z$ is Lipschitz continuous. Thus, $z$ is a Lipschitz continuous solution of the initial value problem for (6.15) with initial values (6.13). From this it follows that the characteristics are uniquely determined in the entire domain of dependence $D$ of $[a, b]$ and, in turn, that the $R_{i}=m_{i} \theta-m_{j} \phi$, and consequently $\theta$ and $\phi$ also, are uniquely determined there.

The foregoing establishes the desired uniqueness in the domain of dependence of largest open interval $I$ on which $0<\theta^{*}(x)<\pi / 2$, so that we only need to consider the case that $I \neq \mathbb{R}$. Thus we assume that $I=(a, b) \neq \mathbb{R}$. Assume first that $I=(a, b) \neq \varnothing, a, b \in \mathbb{R}$. In this case the domain of dependence is the curvilinear triangle made up of $\bar{I}$, an $\operatorname{arc} A$ of the rightmost 1-characteristic 
exiting at $a$, and an $\operatorname{arc} B$ of the leftmost 2-characteristic exiting at $b$. Since the fan angles at $b$ are determined by the size of the jump in $\theta^{*}$ at $b$, we see that an initial piece of the 2-fan at $b$, being determined by the corresponding fan angle and $B$, is unique. Now, the 1-characteristics making up the 1-fan at $b$ emanate from $b$, are concave towards the right and have angles which tend to 0 , and furthermore do not exit to the right of $b$. From this it follows that they are horizontal lines, which gives us the uniqueness of the 1 -fan at $b$ and hence the uniqueness of $\theta$ and $\phi$ in $N([b, \infty), \epsilon)$ for some $\epsilon>0$. (The case in which there is no jump at $b$ is essentially the same, with only minor changes.) Similarly, we get uniqueness in a neighborhood $N([-\infty, a), \epsilon)$. Since $\theta$ and $\phi$ are Lipschitz continuous on all horizontal lines in $H$, their uniqueness in the entire half-plane follows immediately. That $f$ itself is unique follows from the condition $f(0)=0$. The cases in which $a=-\infty$ or $b=\infty$ are in effect included in this argument. The only remaining cases are those in which $(a, b)=\varnothing$, that is, those in which $\theta^{*}$ is identically 0 or $\pi / 2$ on $\mathbb{R}$ or in which $\theta^{*}(x)$ jumps from $\pi / 2$ to 0 at $a \in \mathbb{R}$. In the first two of these cases, $f$ is easily seen to be linear. In the final case, let the angular sizes of the 1- and 2-fans at $a$ be $\delta_{1}$ and $\delta_{2}$, and let the angular sizes of the sectors to the right and left of these fans, respectively, be $\alpha_{R}$ and $\alpha_{L}$. Then, it is clear from Theorem 5.1 that $\alpha_{R}=\alpha_{L}=0$ since if $\alpha_{R}>0$, for example, there would be a 1-characteristic exiting to the right of $a$. As above, on the basis of the concavity of the characteristics, we see that the fans at $a$ are made up of straight lines. The uniqueness then follows since the numbers $\delta_{1}$ and $\delta_{2}$ are uniquely determined by conditions (5.2).

It is clear that the proof can be modified to show uniqueness for solutions to Cauchy problems for (1.2) associated with transformation problems in much greater generality.

7. Closing remarks. In this section we briefly discuss some issues pertinent to further investigation of the transformation problem for cps-mappings. We begin by pointing out that although Theorem 6.9 might lead one to believe that "exterior domains" are somewhat simpler to deal with than Jordan domains, the half-plane situation is, in effect, a fluke because the absence of doubly exiting characteristics essentially (i.e., apart from the simple degenerate cases in which $\theta^{*}(x)=\pi / 2$ on $(-\infty, a)$ and/or 0 on $(b, \infty)$, discussed in the final paragraph of the preceding section) means that all of the mappings in question are reducible to Cauchy problems. It is not too hard to show that this favorable circumstance arises also in the case of cps-self-homeomorphisms of the exterior of a disk, making possible their complete description. This case, though, is substantially more complex than that of half-planes since it involves two fundamentally different classes of mappings, corresponding to the two kinds of HP-nets with a single isolated singularity in $\mathbb{C}$ (see [5, Theorem 3.3]). Moreover, in contrast to the half-plane case in which the Cauchy data is given on a straight line and so (interpretation aside) has the standard initial value format, 
in the case of $\mathbb{C} \backslash N(0,1)$, the data given on $\partial N(0,1)$ actually generates a unique solution in the entire exterior of the disk. Because it would appear that there are few domains for which no cps-self-homeomorphisms have doubly exiting characteristics, it would be of considerable interest to determine all of them; in fact, it may well turn out that the only possibilities are half-planes and the exteriors of disks.

Although the most appealing transformation problems for cps-mappings are ones for which $D$ and $D^{\prime}$ are (piecewise smoothly bounded) Jordan domains, this case unfortunately leads to a highly over-determined situation in which there is no way of working exclusively with Cauchy problems since all of the characteristics are necessarily doubly exiting, and which is further complicated by the possibility of nonexistence established in Theorem 3.1. These circumstances suggest that one seek explicit nonexistence and qualitative results, and in this direction we formulate two conjectures.

CONJECTURE 7.1. There is a very strong tendency for cps-homeomorphisms to bend domains (because of the way they change total curvature of characteristics); specifically, there are no cps-self-homeomorphisms of disks.

CONJECTURE 7.2. For any two given smoothly bounded Jordan domains and any given $m_{1}, m_{2}$ there are at most a finite number of $\left(m_{1}, m_{2}\right)$-homeomorphisms of one onto the other.

We believe that the case of cps-self-homeomorphisms of strips should prove both interesting, because they are "almost" Jordan domains, and tractable, because of the geometric simplicity of their boundaries, even though it is likely that it nonetheless gives rise to subtle blow-up questions.

In [5] we showed that for an HP-function $\theta$ defined on a smoothly bounded Jordan domain $D$ the nontangential limits of $\theta$ exist a.e. on $\partial D$, a conclusion considerably weaker than that of Corollary 5.8. We believe, however, that one can replace the proof of [5] by one that yields the conclusion of this corollary without any assumption beyond the smoothness of $\partial D$. Doing so, however, will require an analysis of the local behavior and global consequences associated with the two kinds of boundary singularities that we have shown cannot arise in the context of a cps-homeomorphism of $D$ onto a smoothly bounded image domain, namely, points $p \in \partial D$ at which there is an exiting characteristic along which $\lim _{z \rightarrow p} \theta(z)$ does not exist and those at which there are simply no exiting characteristics at all, both of which possibilities can present themselves in the more general context.

One can contemplate the extension of some of the results about planar cpsmappings to their higher-dimensional counterparts. In that context, the underlying equations corresponding to (1.2) have in some sense a similar form (see [4]), but are substantially more complex and allow considerably more leeway for the avoidance of singularity formation. In particular, there exist nontrivial cps-self-homeomorphisms of $\mathbb{R}^{3}$, examples of which were found by Yin [14], 
and we believe that an interesting investigation would result from an attempt to discover all of them.

Before closing, we must mention the significant foundational conjecture that the partial derivatives of a $C^{1}$ mapping $f: D \rightarrow \mathbb{C}$ with constant principal strains $m_{1} \neq m_{2}$ are necessarily locally Lipschitz continuous. In this direction we showed in [3] that if $J_{f}$ is Hölder continuous with exponent $\alpha>(1 / 2)(\sqrt{5}-1), f$ is necessarily an $\left(m_{1}, m_{2}\right)$-mapping, and the proof can be sharpened to show that $\alpha>1 / 2$ is sufficient.

\section{REFERENCES}

[1] C. Carathéodory and E. Schmidt, Über die Hencky-Prandtlschen Kurven, Z. angew. Math. Mech. 3 (1923), 468-475 (German).

[2] M. Chuaqui and J. Gevirtz, Constant principal strain mappings on 2-manifolds, SIAM J. Math. Anal. 32 (2000), no. 4, 734-759.

[3] J. Gevirtz, On planar mappings with prescribed principal strains, Arch. Rational Mech. Anal. 117 (1992), no. 4, 295-320.

[4] _ A diagonal hyperbolic system for mappings with prescribed principal strains, J. Math. Anal. Appl. 176 (1993), no. 2, 390-403.

[5] __ Hencky-Prandtl nets and constant principal strain mappings with isolated singularities, Ann. Acad. Sci. Fenn. Math. 25 (2000), no. 1, 187-238.

[6] _ Singularity sets of constant principal strain deformations, J. Math. Anal. Appl. 263 (2001), no. 2, 600-625.

[7] W. S. Hemp, Optimum Structures, Clarendon Press, Oxford, 1973.

[8] H. Hencky, Über einige statisch bestimmte Fälle des Gleichgewichts in plastischen Körpern, Z. angew. Math. Mech. 3 (1923), 241-251 (German).

[9] R. Hill, The Mathematical Theory of Plasticity, Clarendon, Press, Oxford, 1964.

[10] F. John, On quasi-isometric mappings. I, Comm. Pure Appl. Math. 21 (1968), 77110.

[11] _ On quasi-isometric mappings. II, Comm. Pure Appl. Math. 22 (1969), 265278.

[12] C. Pommerenke, Univalent Functions, Studia Mathematica/Mathematische Lehrbücher, vol. 25, Vandenhoeck \& Ruprecht, Göttingen, 1975.

[13] L. Prandtl, Über die Eindringungsfestigkeit (Härte) plastische Baustoffe und die Festigkeit von Schneiden, Z. Angew. Math. Mech. 1 (1921), 15-20 (German).

[14] W.-L. Yin, Two families of finite deformations with constant strain invariants, Mech. Res. Comm. 10 (1983), no. 3, 127-132.

Julian Gevirtz: Facultad de Matemáticas, Pontificia Universidad Católica de Chile, Casilla 306, Santiago 22, Chile and Academia Chilena de Ciencias, Almirante Montt 454, Santiago, Chile

Current address: 2005 North Winthrop Road, Muncie, IN 47304, USA

E-mail address: jgevirtz@bsu.edu 


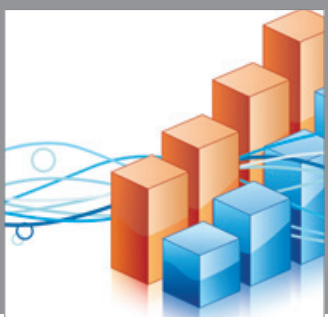

Advances in

Operations Research

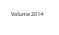

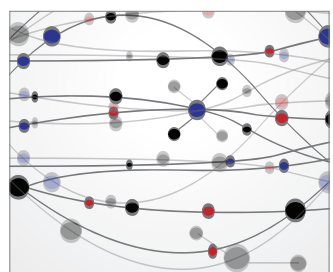

\section{The Scientific} World Journal
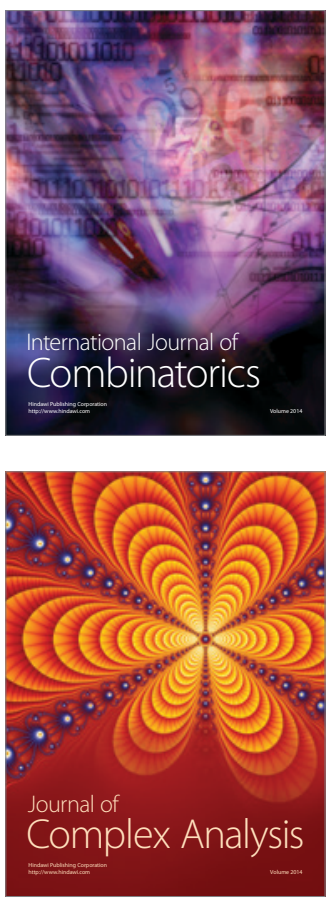

International Journal of

Mathematics and

Mathematical

Sciences
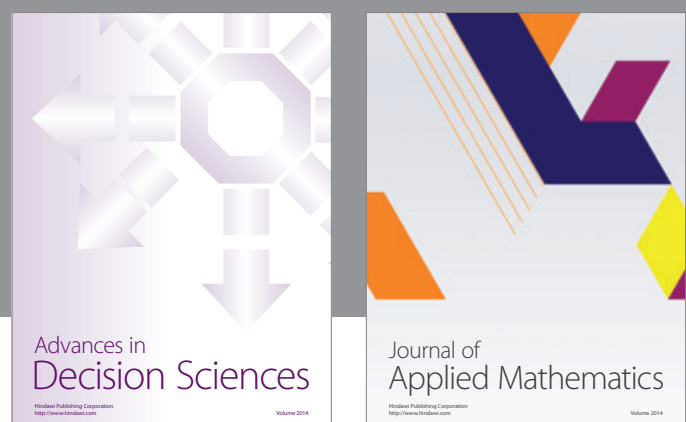

Journal of

Applied Mathematics
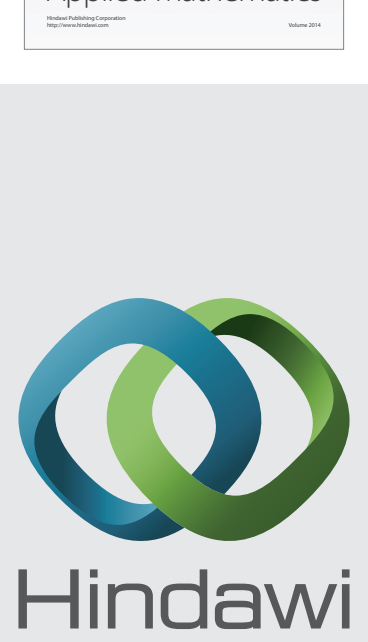

Submit your manuscripts at http://www.hindawi.com
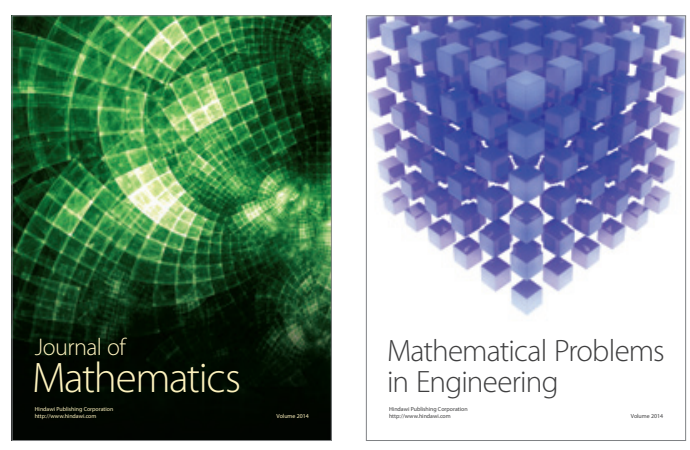

Mathematical Problems in Engineering
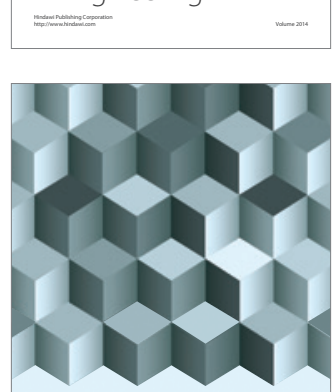

Journal of

Function Spaces
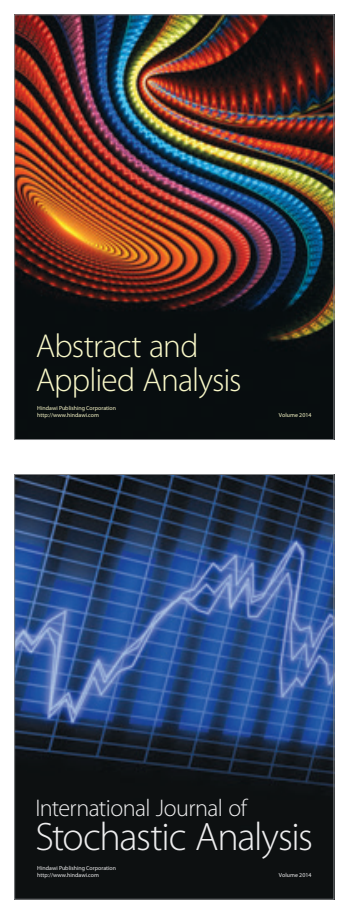

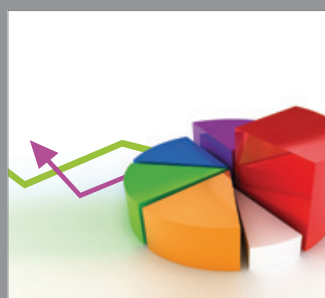

ournal of

Probability and Statistics

Promensencen
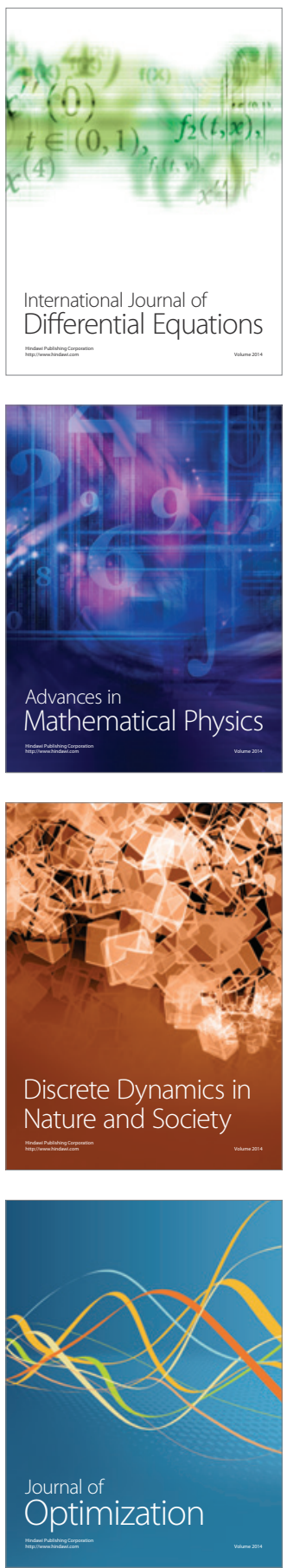